\title{
Long-time behavior of solutions to a fourth-order nonlinear Schrödinger equation with critical nonlinearity
}

\author{
MAMORU OKAMOTOD AND KOTA URIYA
}

Abstract. We consider the long-time behavior of solutions to a fourth-order nonlinear Schrödinger (NLS) equation with a derivative nonlinearity. By using the method of testing by wave packets, we construct an approximate solution and show that the solution for the fourth-order NLS has the same decay estimate for linear solutions. We prove that the self-similar solution is the leading part of the asymptotic behavior.

\section{Introduction}

We consider the Cauchy problem for a fourth-order nonlinear Schrödinger (NLS) equation

$$
\begin{cases}i \partial_{t} u-\frac{1}{4} \partial_{x}^{4} u=i \partial_{x} F(u), & t>0, x \in \mathbb{R} \\ u(0, x)=u_{0}(x), & x \in \mathbb{R},\end{cases}
$$

where $u=u(t, x):[0, \infty) \times \mathbb{R} \rightarrow \mathbb{C}$ is an unknown function and $u_{0}$ is a given function. Here, $F$ satisfies the following assumptions:

A-1. $F \in C^{1}(\mathbb{C} ; \mathbb{C}) \cap C^{2}(\mathbb{C} \backslash\{0\} ; \mathbb{C})^{1}$ with $F(0)=F^{\prime}(0)=0$ and $F(\alpha u)=\alpha^{4} F(u)$ for $\alpha \geq 0$ and $u \in \mathbb{C}$, where $F^{\prime}$ denotes any of $F_{u}:=\frac{\partial F}{\partial u}$ and $F_{\bar{u}}:=\frac{\partial F}{\partial \bar{u}}$. Moreover,

$$
\left|F^{\prime}\left(u_{1}\right)-F^{\prime}\left(u_{2}\right)\right| \lesssim\left(\left|u_{1}\right|^{2}+\left|u_{2}\right|^{2}\right)\left|u_{1}-u_{2}\right|
$$

for all $u_{1}, u_{2} \in \mathbb{C}$.

\section{A-2. $F_{u}$ is real-valued.}

We use the assumption (A-1) to show the local-in-time well-posedness of (1.1). More precisely, we can prove the local well-posedness of (1.1) with the quartic homogeneity replaced by

$$
\left|F^{(j)}(u)\right| \lesssim|u|^{4-j}
$$

for $j=0,1,2$ and $u \neq 0$. However, we only consider the quartic homogeneous nonlinearity in this paper for simplicity. See also Remark 1.

Mathematics Subject Classification: 35Q55, 35B40

Keywords: Fourth-order Schrödinger equation, Asymptotic behavior, Scattering, Self-similar solution. ${ }^{1}$ Here, we regard $\mathbb{C}$ as $\mathbb{R}^{2}$. 
To obtain the global existence (and asymptotic behavior), we employ the quartic homogeneity and (A-2). Indeed, we use these assumptions in energy estimates in Sect. 2. A typical example of $F$ is given by

$$
F(u)=a|u|^{3} u+b \bar{u}^{4}
$$

for $a \in \mathbb{R}$ and $b \in \mathbb{C}$. We note that the first term $|u|^{3} u$ in (1.3) can be generalized as follows: for a real-valued cubic homogeneous function $g \in C^{1}(\mathbb{C} ; \mathbb{R}) \cap C^{2}(\mathbb{C} \backslash\{0\} ; \mathbb{R})$, $\int_{0}^{u} g(v) \mathrm{d} v$ satisfies assumptions (A-1) and (A-2), where we calculate this integral as if $u$ is a real-variable. For example, when $g(u)=|u|^{3}=u^{\frac{3}{2}} \bar{u}^{\frac{3}{2}}$, we have

$$
\int_{0}^{u} g(v) \mathrm{d} v=\frac{2}{5} u^{\frac{5}{2}} \bar{u}^{\frac{3}{2}}=\frac{2}{5}|u|^{3} u .
$$

By setting $g(u)=(\Re u)^{3-k}(\Im u)^{k}$ for $k=0,1,2,3$, we have other examples of nonlinearities satisfying (A-1) and (A-2).

Here, we mention some properties of solutions to (1.1). If $u$ is a solution to (1.1), we have the following conservation law:

$$
\int_{\mathbb{R}} u(t, x) \mathrm{d} x=\int_{\mathbb{R}} u_{0}(x) \mathrm{d} x .
$$

Note that (1.1) is invariant under the scaling transformation

$$
u(t, x) \mapsto \lambda u\left(\lambda^{4} t, \lambda x\right)
$$

for any $\lambda>0$. Hence, the scaling critical Sobolev regularity is $s_{c}:=-\frac{1}{2}$.

Asymptotic behavior of the fourth-order NLS and its related equations have been studied by several researchers. See [1,2,5-12,14,15,19] and references therein. In particular, Ben-Artzi, Koch, and Saut [2] showed the dispersive estimates for the fourth-order Schrödinger equations. From the dispersive estimates, we can expect that a quartic nonlinearity with a derivative is critical in the sense of the asymptotic behavior of solutions to (1.1). This is a reason why we assume quartic nonlinearity in (A-1).

Hayashi and Naumkin [6,7] derived the asymptotic behavior of the solution to the fourth-order NLS equation with the gauge invariant nonlinearity:

$$
i \partial_{t} u-\frac{1}{4} \partial_{x}^{4} u=\lambda \partial_{x}\left(|u|^{\rho} u\right), \quad t>0, x \in \mathbb{R} .
$$

They proved that the asymptotic behavior of (1.6) is the same as that of the linear solution and the self-similar solution to (1.6) when $\lambda \in \mathbb{C}, \rho>3$ and $\lambda=i, \rho=3$, respectively. They employed the factorization technique for the evolution operator of the fourth-order Schrödinger equation.

For (1.1) with $F(u)=\bar{u}^{4}$, namely

$$
i \partial_{t} u-\frac{1}{4} \partial_{x}^{4} u=\partial_{x}\left(\bar{u}^{4}\right), \quad t>0, x \in \mathbb{R},
$$


Hirayama and the first author [12] showed the small data global well-posedness and the scattering in the scaling critical Sobolev space $\dot{H}^{-\frac{1}{2}}(\mathbb{R})$. They used the Fourier restriction norm method adapted to the spaces $V^{p}$ of functions of bounded $p$-variation and their pre-duals $U^{p}$.

To state the main result, we denote $H^{s, r}(\mathbb{R})$ the weighted Sobolev space equipped with the norm

$$
\|u\|_{H^{s, r}}:=\left\|\langle x\rangle^{r}\left\langle i \partial_{x}\right\rangle^{s} u\right\|_{L_{x}^{2}}
$$

for $s, r \in \mathbb{R}$ and we set $H^{s}(\mathbb{R}):=H^{s, 0}(\mathbb{R})$. Define the phase function

$$
\phi(t, x)=\frac{3}{4} t^{-\frac{1}{3}} x^{\frac{4}{3}}-\frac{\pi}{4} .
$$

Here, $a^{\frac{1}{3}}=\sqrt[3]{a}$ denotes the unique real cubic root of $a \in \mathbb{R}$.

Theorem 1. Assume that the initial datum $u_{0}$ at time 0 satisfies

$$
\left\|u_{0}\right\|_{H^{1}}+\left\|u_{0}\right\|_{H^{0,1}} \leq \varepsilon \ll 1 .
$$

Let $F$ satisfy (A-1) and (A-2). Then, there exists a unique global solution $u$ to (1.1) with $e^{i \frac{1}{4} t \partial_{x}^{4}} u \in C\left([0, \infty) ; H^{1}(\mathbb{R}) \cap H^{0,1}(\mathbb{R})\right)$ satisfying the estimates

$$
\left\|\left\langle t^{-\frac{1}{4}} x\right\rangle^{-\frac{k}{3}+\frac{1}{3}} \partial_{x}^{k} u(t)\right\|_{L_{x}^{\infty}} \lesssim \varepsilon t^{-\frac{k+1}{4}}
$$

for $t \geq 1$ and $k=0,1,2$. Moreover, we have the following asymptotic behavior as $t \rightarrow+\infty$.

Set $\rho:=\frac{1}{4}\left(\frac{1}{8}-\varepsilon\right)$. In the self-similar region $\mathfrak{X}^{\text {self }}(t):=\left\{x \in \mathbb{R}: t^{-\frac{1}{4}}|x| \lesssim t^{3 \rho}\right\}$, there exists a solution $Q=Q(y)$ to the nonlinear ordinary differential equation

$$
Q^{\prime \prime \prime}+i y Q+4 i F(Q)=0
$$

satisfying $\|Q\|_{L_{y}^{\infty}} \lesssim \varepsilon$ and

$$
\begin{aligned}
\left\|u(t)-t^{-\frac{1}{4}} Q\left(t^{-\frac{1}{4}} x\right)\right\|_{L_{x}^{\infty}\left(\mathfrak{X}^{\text {self }}(t)\right)} & \lesssim \varepsilon t^{-\frac{1}{4}-\frac{5}{2} \rho}, \\
\left\|u(t)-t^{-\frac{1}{4}} Q\left(t^{-\frac{1}{4}} x\right)\right\|_{L_{x}^{2}\left(\mathfrak{X}^{\text {self }}(t)\right)} & \lesssim \varepsilon t^{-\frac{1}{8}-3 \rho} .
\end{aligned}
$$

In the oscillatory region $\mathfrak{X}^{\mathrm{osc}}(t):=\left\{x \in \mathbb{R}: t^{-\frac{1}{4}}|x| \gtrsim t^{3 \rho}\right\}$, there exists a unique complex-valued function $W$ satisfying $\|W\|_{L^{\infty} \cap L^{2}} \lesssim \varepsilon$ such that

$$
u(t, x)=\frac{1}{\sqrt{3}} t^{-\frac{1}{4}}\left(t^{-\frac{1}{4}} x\right)^{-\frac{1}{3}} W\left(t^{-\frac{1}{3}} x^{\frac{1}{3}}\right) e^{i \phi(t, x)}+\mathbf{e r r}_{x},
$$

where the error satisfies the estimates

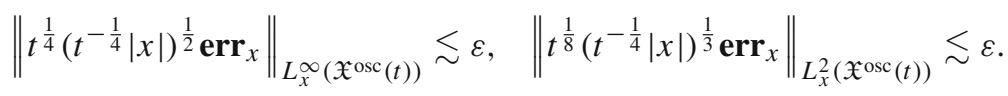


In the corresponding frequency region $\widehat{\mathfrak{X}}^{\mathrm{osc}}(t):=\left\{\xi \in \mathbb{R}: t^{\frac{1}{4}}|\xi| \gtrsim t^{\rho}\right\}$, we have

$$
\widehat{u}(t, \xi)=W(\xi) e^{\frac{1}{4} i t \xi^{4}}+\operatorname{err}_{\xi},
$$

where the error satisfies

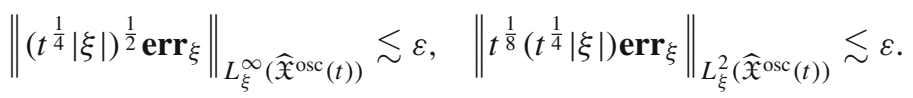

In Theorem 1 , we divide $\mathbb{R}$ into two regions $\mathbb{R}=\mathfrak{X}^{\text {self }}(t) \cup \mathfrak{X}^{\text {osc }}(t)$. Note that, in the results on $\mathrm{KdV}$ equations in $[3,17,18]$, the asymptotic behavior is classified into three regions: self-similar, oscillatory, and decaying. This difference comes from the asymptotic behavior of the linear solutions. Indeed, the corresponding linear equation to $(1.1)$

$$
i \partial_{t} u-\frac{1}{4} \partial_{x}^{4} u=0
$$

is invariant under the spatial inversion. Namely, if $u$ satisfies (1.15), then $\tilde{u}$ defined by

$$
\widetilde{u}(t, x):=u(t,-x)
$$

also satisfies the same equation. Hence, the asymptotic behaviors for $x>0$ and $x<0$ are the same. On the other hand, the linear KdV (Airy) equation

$$
\partial_{t} u-\frac{1}{3} \partial_{x}^{3} u=0
$$

is not invariant under the spatial inversion (1.16). More precisely, the transformation (1.16) changes the sign of the coefficient of $\partial_{x}^{3}$. Indeed, the solution to (1.17) (the Airy function) is oscillating for $x>0$ and decaying for $x<0$.

As mentioned above, by using the factorization technique for the fourth-order NLS equation, Hayashi and Naumkin [6] studied the asymptotic behavior of (1.1) with $F(u)=|u|^{3} u$ for small initial data in $H^{1,1}(\mathbb{R})$. More precisely, they proved the existence of a global solution $u$ with $e^{i \frac{1}{4} t \partial_{x}^{4}} u \in C\left([0, \infty) ; H^{1,1}(\mathbb{R})\right)$ and

$$
\|u(t)\|_{L_{x}^{\infty}} \lesssim \varepsilon\langle t\rangle^{-\frac{1}{4}}
$$

when $\left\|u_{0}\right\|_{H^{1,1}} \leq \varepsilon \ll 1$. In this paper, we employ the method of testing by wave packets as in [13]. Since we use (1.9) instead of (1.18) (as a bootstrap assumption), our assumption $u_{0} \in H^{1}(\mathbb{R}) \cap H^{0,1}(\mathbb{R})$ is better than $u_{0} \in H^{1,1}(\mathbb{R})$ in [6]. See also Remark 2.

Remark 1. We can obtain the same result as in Theorem 1 for short-range perturbations of the form

$$
i \partial_{t} u-\frac{1}{4} \partial_{x}^{4} u=i \partial_{x}(F(u)+G(u)),
$$


where $G \in C^{2}(\mathbb{C} ; \mathbb{C}), G_{u}$ is real-valued, and there exists $p_{0}>4$ such that

$$
\left|G^{(j)}(u)\right| \lesssim|u|^{p_{0}-j}
$$

for $j=0,1,2$. Since we can apply the same argument as in Appendix A in [3] and Appendix B in [18], we omit the details here.

Remark 2. When we consider the explicit nonlinearity as in (1.3), we can replace $H^{1}(\mathbb{R})$ in Theorem 1 with $H^{\frac{3}{8}}(\mathbb{R})$. See Remark 4 . Note that this regularity $H^{\frac{3}{8}}(\mathbb{R})$ is exactly the same as that in [18] with the fourth-order dispersion.

\subsection{Outline of proof}

We give here an outline of the proof. Denote by $\mathcal{L}$ the linear operator of (1.1):

$$
\mathcal{L}:=i \partial_{t}-\frac{1}{4} \partial_{x}^{4}
$$

To obtain pointwise estimates for solutions, we use the vector field

$$
\mathcal{J}:=x-i t \partial_{x}^{3},
$$

which satisfies $\mathcal{J}=e^{-i \frac{1}{4} t \partial_{x}^{4}} x e^{i \frac{1}{4} t \partial_{x}^{4}}$. Since $\mathcal{J}$ has the third derivative, it is difficult to apply $\mathcal{J}$ directly for the energy estimates. We then use the generator of the scaling transformation (1.5) given by

$$
\mathcal{S}:=4 t \partial_{t}+x \partial_{x}+1
$$

Moreover, by (1.19)-(1.21), we have

$$
\mathcal{S}=-4 i t \mathcal{L}+\mathcal{J} \partial_{x}+1 .
$$

As in $[3,17,18]$, we also use the operator

$$
\Lambda:=\partial_{x}^{-1} \mathcal{S}=-4 i t \partial_{x}^{-1} \mathcal{L}+\mathcal{J} .
$$

Roughly speaking, since the operator $\Lambda$ acts as the first-order derivative for the nonlinearity, we use $\Lambda$ instead of $\mathcal{J}$.

We introduce the norm with respect to the spatial variable

$$
\|u(t)\|_{X}:=\left(\|u(t)\|_{H_{x}^{1}}^{2}+\|\Lambda u(t)\|_{L_{x}^{2}}^{2}\right)^{\frac{1}{2}} .
$$

We note that

$$
\left\|u_{0}\right\|_{X} \sim\left\|u_{0}\right\|_{H^{1}}+\left\|u_{0}\right\|_{H^{0,1}} .
$$

By a standard fixed point argument, we have the local well-posedness in $X$ of (1.1). 
Proposition 1. Assume that $F$ satisfies $(A-1)$. If $u_{0} \in H^{1}(\mathbb{R}) \cap H^{0,1}(\mathbb{R})$ satisfies (1.8), then there exist $T>1$ and a (unique) solution $u(t) \in X$ to (1.1) satisfying

$$
\sup _{0 \leq t \leq T}\|u(t)\|_{X} \lesssim\left\|u_{0}\right\|_{H^{1}}+\left\|u_{0}\right\|_{H^{0,1}} .
$$

The proof is a slight modification of that in Appendix in [18].

We then make the bootstrap assumption that $u$ satisfies the linear pointwise estimates: there exists a large constant $D$ such that

$$
\left\|\left\langle t^{-\frac{1}{4}} x\right\rangle^{-\frac{k}{3}+\frac{1}{3}} \partial_{x}^{k} u(t)\right\|_{L_{x}^{\infty}} \leq D \varepsilon t^{-\frac{k+1}{4}}
$$

for $t \in[1, T]$ and $k=0,1,2$. Note that we take $\varepsilon>0$ small enough so that $\varepsilon \leq D^{-2}$.

In Sect. 2, by using (1.26), for $\varepsilon>0$ sufficiently small, we prove the a priori bound:

$$
\sup _{1 \leq t \leq T}\|u(t)\|_{X} \leq \varepsilon C_{T},
$$

where $C_{T}$ is a constant depending only on $T$. Namely, $C_{T}$ is independent of $D$ and $\varepsilon$. Then, by the local well-posedness with (1.27), the global existence follows from closing the bootstrap estimate (1.26).

In Sect. 3, we prove decay estimates in $L^{\infty}(\mathbb{R})$ and $L^{2}(\mathbb{R})$ that allow us to reduce closing the bootstrap argument to considering the behavior of $u$ along the ray $\Gamma_{v}:=$ $\{x=v t\}$. We also observe that (1.26) holds true at $t=1$. Since $u$ is complex-valued, we have to pay attention to the sign of frequencies. We thus need to slightly modify the argument in [18]. See, for example, (3.11) and the proof of Lemma 4.

To close the bootstrap argument, we use the method of testing by wave packets as in $[3,4,13,18]$. Here, a wave packet is an approximate solution localized in both space and frequency on the scale of the uncertainty principle. Our main task in Sect. 4 is to construct a wave packet $\Psi_{v}(t, x)$ to the corresponding linear equation and observe its properties.

To observe decay of $u$ along the ray $\Gamma_{v}$, we use the function

$$
\gamma(t, v)=\int_{\mathbb{R}} u(t, x) \overline{\Psi_{v}(t, x)} \mathrm{d} x .
$$

In Sect. 4, we prove that $\gamma$ is a reasonable approximation of $u$. We then reduce closing the bootstrap estimate (1.26) to proving global bounds for $\gamma$.

In Sect. 5, by solving an ordinary differential equation with respect to $\gamma$, we show the global existence of $u$. Moreover, we prove that the leading part of the asymptotic behavior is given by the self-similar solution $t^{-\frac{1}{4}} Q\left(t^{-\frac{1}{4}} x\right)$, where $Q$ is a solution to (1.10).

\subsection{Notation}

At this point, we summarize the notation used throughout this paper. Set $\mathbb{N}_{0}:=\mathbb{N} U$ $\{0\}$. Denote the set of positive and negative real numbers by $\mathbb{R}_{+}$and $\mathbb{R}_{-}$, respectively. 
Let $C_{0}^{\infty}(\mathbb{R})$ be the space of all smooth and compactly supported functions. We denote the space of all smooth and rapidly decaying functions on $\mathbb{R}$ by $\mathcal{S}(\mathbb{R})$. Define the Fourier transform of $f$ by $\mathcal{F}[f]$ or $\widehat{f}$.

In estimates, we use $C$ to denote a positive constant that can change from line to line. If $C$ is absolute or depends only on parameters that are fixed, then we often write $X \lesssim Y$, which means $X \leq C Y$. When an implicit constant depends on a parameter $a$, we sometimes write $X \lesssim a Y$. We define $X \ll Y$ to mean $X \leq C^{-1} Y$ and $X \sim Y$ to mean $C^{-1} Y \leq X \leq C Y$. We write $X=Y+O(Z)$ when $|X-Y| \lesssim Z$.

Let $\sigma$ be a smooth even function with $0 \leq \sigma \leq 1$ and

$$
\sigma(\xi)= \begin{cases}1, & \text { if }|\xi| \leq 1 \\ 0, & \text { if }|\xi| \geq 2\end{cases}
$$

For any $R, R_{1}, R_{2}>0$ with $R_{1}<R_{2}$, we set

$$
\begin{array}{r}
\sigma_{\leq R}(\xi):=\sigma\left(\frac{\xi}{R}\right), \quad \sigma_{>R}(\xi):=1-\sigma_{\leq R}(\xi), \\
\sigma_{<R}(\xi):=\sigma_{\leq \frac{R}{2}}(\xi), \quad \sigma_{\geq R}(\xi):=1-\sigma_{<R}(\xi), \quad \sigma_{R}(\xi):=\sigma_{\leq R}(\xi)-\sigma_{<R}(\xi), \\
\sigma_{R_{1} \leq . \leq R_{2}}(\xi):=\sigma_{\leq R_{2}}(\xi)-\sigma_{<R_{1}}(\xi), \quad \sigma_{R_{1}<\cdot<R_{2}}(\xi):=\sigma_{<R_{2}}(\xi)-\sigma_{\leq R_{1}}(\xi) .
\end{array}
$$

Moreover, we define the corresponding Fourier multipliers as usual:

$$
\begin{aligned}
& P_{R} f:=\mathcal{F}^{-1}\left[\sigma_{R} \widehat{f}\right], \quad P_{\leq R} f:=\mathcal{F}^{-1}\left[\sigma_{\leq R} \widehat{f}\right], \quad P_{>R} f:=\mathcal{F}^{-1}\left[\sigma_{>R} \widehat{f}\right], \\
& P_{R_{1} \leq \leq R_{2}} f:=\mathcal{F}^{-1}\left[\sigma_{R_{1} \leq \cdot \leq R_{2}} \widehat{f}\right] .
\end{aligned}
$$

We denote the characteristic function of an interval $I$ by $\mathbf{1}_{I}$. For $N \in 2^{\mathbb{Z}}$, we define

$$
P^{ \pm} f:=\mathcal{F}^{-1}\left[\mathbf{1}_{\mathbb{R}_{ \pm}} \widehat{f]}, \quad P_{N}^{ \pm}:=P^{ \pm} P_{N} .\right.
$$

We also set $\sigma^{ \pm}=\sigma \mathbf{1}_{\mathbb{R}^{ \pm}}$and $\sigma_{\leq R}^{ \pm}:=\sigma_{\leq R} \mathbf{1}_{\mathbb{R}^{ \pm}}$, etc.

\section{Energy estimates}

In this section, we prove some a priori estimates of a solution $u$ to (1.1) satisfying (1.26). First, we use an energy estimate to obtain the bound for $\|u(t)\|_{X}$.

Lemma 1. Assume that $F$ satisfies (A-1) and (A-2). Let $u$ be a solution to (1.1) in a time interval $[0, T]$ satisfying

$$
\left\|u_{0}\right\|_{H^{1}}+\left\|u_{0}\right\|_{H^{0,1}} \leq \varepsilon \ll 1
$$

and (1.26). Then, we have

$$
\|u(t)\|_{X} \lesssim \varepsilon\langle t\rangle^{\varepsilon},
$$

where $X$ is defined in (1.23) and the implicit constant is independent of $D, T$, and $\varepsilon$. 
Proof. By (1.25), we have the desired bound for $0 \leq t \leq 1$. We thus consider the case $t>1$.

It follows from (1.1) and (A-1) that

$$
\begin{aligned}
\frac{1}{2} \frac{\mathrm{d}}{\mathrm{d} t}\|u(t)\|_{L_{x}^{2}}^{2} & =\Re \int_{\mathbb{R}} u \cdot\left(\overline{F_{u}(u) \overline{\partial_{x}} u}+\overline{F_{\bar{u}}(u)} \partial_{x} u\right) \mathrm{d} x \\
& \lesssim\|u(t)\|_{L_{x}^{2}}^{2}\|u(t)\|_{L_{x}^{\infty}}^{2}\left\|\partial_{x} u(t)\right\|_{L_{x}^{\infty}}
\end{aligned}
$$

By (1.1) and (1.2), we have

$$
\begin{aligned}
\frac{1}{2} \frac{\mathrm{d}}{\mathrm{d} t}\left\|\partial_{x} u(t)\right\|_{L_{x}^{2}}^{2}= & \Re \int_{\mathbb{R}} \partial_{x} u \cdot \overline{F_{u}(u)} \overline{\partial_{x}^{2} u} \mathrm{~d} x+\Re \int_{\mathbb{R}} \partial_{x} u \cdot \overline{F_{\bar{u}}(u)} \partial_{x}^{2} u \mathrm{~d} x \\
& +O\left(\|u(t)\|_{H_{x}^{1}}^{2}\|u(t)\|_{L_{x}^{\infty}}^{2}\left\|\partial_{x} u(t)\right\|_{L_{x}^{\infty}}\right) \\
= & : \mathrm{I}+\mathrm{II}+O\left(\|u(t)\|_{H_{x}^{1}}^{2}\|u(t)\|_{L_{x}^{\infty}}^{2}\left\|\partial_{x} u(t)\right\|_{L_{x}^{\infty}}\right) .
\end{aligned}
$$

From $F_{u}(0)=F_{\bar{u}}(0)=0$, we may regard the integrals in I and II as those on $\{u \neq 0\}$. It follows from (A-2), integrating by parts, and (1.2) that

$$
\mathrm{I}=-\frac{1}{2} \int_{\mathbb{R}} \partial_{x} F_{u}(u)\left|\partial_{x} u\right|^{2} \mathrm{~d} x \lesssim\|u(t)\|_{H_{x}^{1}}^{2}\|u(t)\|_{L_{x}^{\infty}}^{2}\left\|\partial_{x} u(t)\right\|_{L_{x}^{\infty}} .
$$

Moreover, we apply integration by parts with (1.2) to obtain

$$
\mathrm{II}=-\frac{1}{2} \Re \int_{\mathbb{R}} \partial_{x} \overline{F_{\bar{u}}(u)}\left(\partial_{x} u\right)^{2} \mathrm{~d} x \lesssim\|u(t)\|_{H_{x}^{1}}^{2}\|u(t)\|_{L_{x}^{\infty}}^{2}\left\|\partial_{x} u(t)\right\|_{L_{x}^{\infty}} .
$$

By (2.2)-(2.5), we obtain

$$
\frac{\mathrm{d}}{\mathrm{d} t}\|u(t)\|_{H_{x}^{1}}^{2} \lesssim\|u(t)\|_{H_{x}^{1}}^{2}\|u(t)\|_{L_{x}^{\infty}}^{2}\left\|\partial_{x} u(t)\right\|_{L_{x}^{\infty}} .
$$

A direct calculation with (1.19) and (1.21) yields that

$$
[\mathcal{L}, \mathcal{S}]=4 \mathcal{L}, \quad\left[\mathcal{S}, \partial_{x}\right]=-\partial_{x}
$$

Moreover, it follows from (A-1) that

$$
4 F(u)=F_{u}(u) u+F_{\bar{u}}(u) \bar{u} .
$$

If $u$ is a solution to (1.1), it follows (1.22) and (1.1) that

$$
\mathcal{L} \Lambda u=\partial_{x}^{-1}(\mathcal{S}+4) \mathcal{L} u=i\left(F_{u}(u) \partial_{x} \Lambda u+F_{\bar{u}}(u) \overline{\partial_{x} \Lambda u}\right) .
$$

By (1.19), (2.7), (A-2), integrating by parts, and (1.2), we obtain

$$
\begin{aligned}
\frac{1}{2} \frac{\mathrm{d}}{\mathrm{d} t}\|\Lambda u(t)\|_{L_{x}^{2}}^{2} & =-\Im \int_{\mathbb{R}} \Lambda u \cdot \overline{\mathcal{L} \Lambda u} \mathrm{~d} x \\
& =-\frac{1}{2} \int_{\mathbb{R}} \partial_{x} F_{u}(u)|\Lambda u|^{2} \mathrm{~d} x-\frac{1}{2} \Re \int_{\mathbb{R}} \partial_{x} \overline{F_{\bar{u}}(u)}(\Lambda u)^{2} \mathrm{~d} x \\
& \lesssim\|\Lambda u(t)\|_{L_{x}^{2}}^{2}\|u(t)\|_{L_{x}^{\infty}}^{2}\left\|\partial_{x} u(t)\right\|_{L_{x}^{\infty}}
\end{aligned}
$$


Hence, it follows from (1.23), (2.6), (2.8), and (1.26) that

$$
\frac{\mathrm{d}}{\mathrm{d} t}\|u(t)\|_{X}^{2} \lesssim(D \varepsilon)^{3} t^{-1}\|u(t)\|_{X}^{2} .
$$

From $(D \varepsilon)^{3} \ll \varepsilon$ and Gronwall's inequality, we obtain

$$
\|u(t)\|_{X} \leq 10\|u(1)\|_{X} \cdot t^{\varepsilon} \lesssim \varepsilon t^{\varepsilon}
$$

for $t \geq 1$.

Remark 3. To obtain (2.6) in the proof of Lemma 1, we only use (1.2) (instead of the quartic homogeneity). However, (2.7) is a consequence of (A-1), and we rely on (A-1) in the calculation in (2.8).

Second, we prove a priori bound for $\|\mathcal{J} u(t)\|_{L_{x}^{2}}$. We define the auxiliary space

$$
\|u(t)\|_{\tilde{X}}:=\|\mathcal{J} u(t)\|_{L_{x}^{2}}+t^{\frac{1}{4}}\left\|\left\langle t^{\frac{1}{4}} \partial_{x}\right\rangle^{-1} u(t)\right\|_{L_{x}^{2}},
$$

where $\mathcal{J}$ is defined in (1.20).

Lemma 2. Assume that $F$ satisfies (A-1) and (A-2). Let $u$ be a solution to (1.1) which satisfies (2.1) and (1.26). Then, for $t \geq 1$, we have

$$
\|u(t)\|_{\widetilde{X}} \lesssim \varepsilon t^{\frac{1}{8}}
$$

where the implicit constant is independent of $D, T$, and $\varepsilon$.

Proof. We note that (1.22) and (1.1) imply that

$$
\mathcal{J} u=\Lambda u+4 i t \partial_{x}^{-1} \mathcal{L} u=\Lambda u-4 t F(u) .
$$

Since (A-1) and (1.26) yield that

$$
|F(u(t, x))| \lesssim|u(t, x)|^{4} \leq \varepsilon t^{-1}\left\langle t^{-\frac{1}{4}} x\right\rangle^{-\frac{4}{3}},
$$

we have

$$
\begin{aligned}
\|F(u(t))\|_{L_{x}^{2}} & \lesssim \varepsilon t^{-1}\left(\int_{t^{-\frac{1}{4}}|x| \leq 1}\left\langle t^{-\frac{1}{4}} x\right\rangle^{-\frac{8}{3}} \mathrm{~d} x+\int_{t^{-\frac{1}{4}}|x| \geq 1}\left\langle t^{-\frac{1}{4}}|x|\right\rangle^{-\frac{8}{3}} \mathrm{~d} x\right)^{\frac{1}{2}} \\
& \lesssim \varepsilon t^{-1+\frac{1}{8}}
\end{aligned}
$$

It follows from (2.10), Lemma 1, and (2.11) that

$$
\|\mathcal{J} u(t)\|_{L_{x}^{2}} \lesssim\|\Lambda u(t)\|_{L_{x}^{2}}+t\|F(u(t))\|_{L_{x}^{2}} \lesssim \varepsilon t^{\varepsilon}+\varepsilon t^{\frac{1}{8}} \lesssim \varepsilon t^{\frac{1}{8}} .
$$

Next, we use a self-similar change of variables by defining

$$
U(t, y):=t^{\frac{1}{4}} u\left(t, t^{\frac{1}{4}} y\right) .
$$


A direct calculation with (1.21) and (1.22) shows

$$
\partial_{t} U(t, y)=\frac{1}{4} t^{-\frac{3}{4}}(\mathcal{S} u)\left(t, t^{\frac{1}{4}} y\right)=\frac{1}{4} t^{-1} \partial_{y}\left((\Lambda u)\left(t, t^{\frac{1}{4}} y\right)\right) .
$$

Then, it follows from (2.14) and Lemma 1 that

$$
\frac{\mathrm{d}}{\mathrm{d} t}\left\|\left\langle\partial_{y}\right\rangle^{-1} U(t)\right\|_{L_{y}^{2}} \lesssim t^{-1-\frac{1}{8}}\|\Lambda u(t)\|_{L_{x}^{2}} \lesssim \varepsilon t^{-1-\frac{1}{8}+\varepsilon} .
$$

By (2.15), taking $0<\varepsilon \ll 1$, and (1.25), we have

$$
\begin{aligned}
\left\|\left\langle\partial_{y}\right\rangle^{-1} U(t)\right\|_{L_{y}^{2}} & =\left\|\left\langle\partial_{y}\right\rangle^{-1} U(1)\right\|_{L_{y}^{2}}+\int_{1}^{t} \partial_{t^{\prime}}\left\|\left\langle\partial_{y}\right\rangle^{-1} U\left(t^{\prime}\right)\right\|_{L_{y}^{2}} \mathrm{~d} t^{\prime} \\
& \lesssim\|u(1)\|_{H_{x}^{-1}}+\varepsilon \lesssim \varepsilon
\end{aligned}
$$

for $t \geq 1$. From $\left\|\left\langle\partial_{y}\right\rangle^{-1} U(t)\right\|_{L_{y}^{2}}=t^{\frac{1}{8}}\left\|\left\langle t^{\frac{1}{4}} \partial_{x}\right\rangle^{-1} u(t)\right\|_{L_{x}^{2}}$, the desired bound follows from (2.12) and (2.16).

Remark 4. The estimate $\|u(t)\|_{\widetilde{X}} \lesssim \varepsilon$ for $0 \leq t \leq 1$ holds true. Indeed, it follows from (2.9), (2.10), (1.23), and Sobolev embedding $H^{\frac{3}{8}}(\mathbb{R}) \hookrightarrow L^{8}(\mathbb{R})$ that

$$
\begin{aligned}
\sup _{0 \leq t \leq 1}\|u(t)\|_{\tilde{X}} & \lesssim \sup _{0 \leq t \leq 1}\left(\|\Lambda u(t)\|_{L_{x}^{2}}+\left\|u(t)^{4}\right\|_{L_{x}^{2}}+\|u(t)\|_{L_{x}^{2}}\right) \\
& \lesssim \sup _{0 \leq t \leq 1}\left(\|u(t)\|_{X}+\|u(t)\|_{X}^{4}\right) .
\end{aligned}
$$

By (1.25), (1.24), and (1.8), we obtain

$$
\sup _{0 \leq t \leq 1}\|u(t)\|_{\tilde{X}} \lesssim \varepsilon
$$

\section{Decay estimates}

In this section, we prove decay estimates for $u$ without the bootstrap assumption (1.26). In Sect. 3.1, we decompose $u$ into a part on which $\mathcal{J}$ acts hyperbolically and a part on which it acts elliptically. Since $u$ is complex-valued, the decomposition is (a bit) different from the previous papers $[3,17,18]$. In Sect. 3.2, by using the decomposition in Sect. 3.1, we prove some decay estimates for $u$.

\subsection{Hyperbolic and elliptic parts of $u$}

We write $u_{N}:=P_{N} u$. Let $N(t) \in 2^{\mathbb{Z}}$ be the smallest dyadic integer satisfying $N(t) \geq t^{-\frac{1}{4}}$ for $t \geq 1$. By setting

$$
u_{<t^{-\frac{1}{4}}}:=P_{<N(t)} u,
$$


we have

$$
u=u_{<t^{-\frac{1}{4}}}+\sum_{\substack{N \in 2^{\mathbb{Z}} \\ N \geq t^{-\frac{1}{4}}}} u_{N} .
$$

Here, by (1.20), we have $\mathcal{J} u_{N}=P_{N}(\mathcal{J} u)+i N^{-1} \mathcal{F}_{\xi}^{-1}\left[\sigma^{\prime}\left(\frac{\xi}{N}\right) \widehat{u}\right]$, where $\sigma^{\prime}$ is a derivative of $\sigma$. Hence, it follows from (2.9) and (3.1) that

$$
\|u(t)\|_{\tilde{X}} \sim\left(\left\|u_{<t^{-\frac{1}{4}}}(t)\right\|_{\widetilde{X}}^{2}+\sum_{\substack{N \in 2^{\mathbb{Z}} \\ N \geq t^{-\frac{1}{4}}}}\left\|u_{N}(t)\right\|_{\widetilde{X}}^{2}\right)^{\frac{1}{2}} .
$$

We decompose $u_{N}$ into positive and negative frequencies:

$$
u_{N}=u_{N}^{+}+u_{N}^{-}, \quad u_{N}^{ \pm}:=P^{ \pm} u_{N}=P_{N}^{ \pm} u
$$

For $t \geq 1$ and $N \geq t^{-\frac{1}{4}}$, we define the hyperbolic and elliptic parts of $u_{N}^{ \pm}$as follows:

$$
u_{N}^{\text {hyp }, \pm}:=\sigma_{N}^{\text {hyp }, \pm} u_{N}^{ \pm}, \quad u_{N}^{\text {ell, } \pm}:=u_{N}^{ \pm}-u_{N}^{\text {hyp }, \pm},
$$

where $\sigma_{N}^{\text {hyp }, \pm}(t, x):=\sigma_{\frac{1}{\kappa} t N^{3} \leq \cdot \leq \kappa t N^{3}}(x) \mathbf{1}_{\mathbb{R}_{ \pm}}(x)$ and

$$
\kappa:=2^{10}
$$

The largeness of $\kappa$ uses in the proof of (3.13) in Lemma 4. While the explicit value of $\kappa$ is not important (e.g., we can choose $\kappa$ with $\kappa \geq 2^{10}$ ), we fix $\kappa$ as in (3.4) for simplicity.

Next, we define

$$
\begin{aligned}
u^{\text {hyp }, \pm}:=\sum_{\substack{N \in 2^{\mathbb{Z}} \\
N \geq t^{-\frac{1}{4}}}} u_{N}^{\text {hyp }, \pm}, \quad u^{\text {hyp }}:=u^{\text {hyp },+}+u^{\text {hyp },-}, \\
u^{\text {ell }}:=u-u^{\text {hyp }} .
\end{aligned}
$$

We note that $u^{\text {hyp, }} \pm$ is supported in $\left\{x \in \mathbb{R}_{ \pm}: t^{-\frac{1}{4}}|x| \geq \frac{1}{2 \kappa}\right\}$. For $(t, x) \in \mathbb{R}^{2}$ with $t^{-\frac{1}{4}}|x| \geq \frac{1}{2 \kappa},(3.4)$ yields that

$$
\#\left\{N \in 2^{\mathbb{Z}}: \frac{1}{2 \kappa} t N^{3} \leq|x| \leq 2 \kappa t N^{3}\right\}<10 .
$$

Hence, $u^{\text {hyp, } \pm}(t, x)$ is a finite sum of $u_{N}^{\text {hyp, } \pm}(t, x)$ 's.

Moreover, we set

$$
u_{N}^{\mathrm{ell}}:=u_{N}^{\mathrm{ell},+}+u_{N}^{\mathrm{ell},-}
$$


for simplicity. It follows from (3.1), (3.3), and (3.6) that

$$
u^{\mathrm{ell}}=u_{<t^{-\frac{1}{4}}}+\sum_{\substack{N \in 2^{\mathbb{Z}} \\ N \geq t^{-\frac{1}{4}}}} u_{N}^{\mathrm{ell}} .
$$

The functions $u_{N}^{\text {hyp }}$ and $u_{N}^{\text {ell }}$ are essentially frequency localized near $N$. This is a consequence of the following lemma. See Lemma 3.1 in [16] and Lemma 4.1 [17] for the proof.

Lemma 3. Let $2 \leq p \leq \infty, N \in 2^{\mathbb{Z}}$, and $R>0$. For any $a, b, c \in \mathbb{R}$ with $a \geq 0$ and $a+c \geq 0$, we have

$$
\left\|\left(1-P_{\frac{N}{2} \leq \leq 2 N}\right)\left|\partial_{x}\right|^{a}\left(|x|^{b} \sigma_{R} P_{N} f\right)\right\|_{L_{x}^{p}} \lesssim a, b, c N^{-c+\frac{1}{2}-\frac{1}{p}} R^{-a+b-c}\left\|P_{N} f\right\|_{L_{x}^{2}} .
$$

Moreover, we may replace $\sigma_{R}$ on the left-hand side by $\sigma_{>R}$ if $a+c>b+1$ and $\sigma_{<R}$ if $a+c \geq 0$ and $b=0$.

In addition, for any $0<r<R$, we have

$$
\begin{aligned}
& \left\|\left(1-P_{\frac{N}{2} \leq-\leq 2 N}\right)\left|\partial_{x}\right|^{a}\left(|x|^{b} \sigma_{r<\cdot<R} P_{N} f\right)\right\|_{L_{x}^{2}} \\
& \quad \lesssim a, b, c N^{-c} R^{-a+b-c}\left(\frac{R}{r}\right)^{a+|b|+c+2}\left\|P_{N} f\right\|_{L_{x}^{2}}
\end{aligned}
$$

Lemma 3 yields that for any $a \geq 0, b \in \mathbb{R}$, and $c \geq 0$,

$$
\begin{gathered}
\left\|\left(1-P_{\frac{N}{2} \leq \leq 2 N}^{ \pm}\right)\left|\partial_{x}\right|^{a}\left(|x|^{b} u_{N}^{\mathrm{hyp}, \pm}(t)\right)\right\|_{L_{x}^{2}} \lesssim_{a, b, c} t^{-\frac{a-b}{4}}\left(t^{\frac{1}{4}} N\right)^{-c}\left\|u_{N}(t)\right\|_{L_{x}^{2}}, \\
\left\|\left(1-P_{\frac{N}{2} \leq \leq \leq 2 N}^{ \pm}\right)\left|\partial_{x}\right|^{a} u_{N}^{\mathrm{ell}, \pm}(t)\right\|_{L_{x}^{2}} \lesssim_{a, c} t^{-\frac{a}{4}}\left(t^{\frac{1}{4}} N\right)^{-c}\left\|u_{N}(t)\right\|_{L_{x}^{2}}, \\
\left\|\left(1-P_{\frac{N}{2} \leq-\leq 2 N}^{ \pm}\right)\left|\partial_{x}\right|^{a}\left(|x|^{b} \sigma_{>t^{\frac{1}{4}}}(x) u_{N}^{\mathrm{ell}, \pm}\right)(t)\right\|_{L_{x}^{2}} \\
\lesssim_{a, b, c} t^{-\frac{a-b}{4}}\left(t^{\frac{1}{4}} N\right)^{-c}\left\|u_{N}(t)\right\|_{L_{x}^{2}} .
\end{gathered}
$$

Factorizing the symbol $x-t \xi^{3}$ of $\mathcal{J}$, we define

$$
\mathcal{J}_{ \pm}:=|x|^{\frac{1}{3}} \pm i t^{\frac{1}{3}} \partial_{x}, \quad \tilde{\mathcal{J}}_{ \pm}:=|x|^{\frac{2}{3}} \mp i t^{\frac{1}{3}}|x|^{\frac{1}{3}} \partial_{x}-t^{\frac{2}{3}} \partial_{x}^{2} .
$$

These operators are useful in our analysis. Note that $\mathcal{J}_{-}$and $\mathcal{J}_{+}$are elliptic on positive and negative frequencies, respectively.

\subsection{Decay estimates in $L^{2}$ and $L^{\infty}$}

First, we show the following frequency localized estimates.

Lemma 4. For $t \geq 1$ and $N \in 2^{\mathbb{Z}}$ with $N \geq t^{-\frac{1}{4}}$, we have

$$
\begin{aligned}
& \left\|\left(|x|^{\frac{2}{3}}+t^{\frac{2}{3}} N^{2}\right) \mathcal{J}_{ \pm} u_{N}^{\text {hyp }, \pm}(t)\right\|_{L_{x}^{2}} \lesssim\left\|u_{N}(t)\right\|_{\tilde{X}}, \\
& \left\|\left(|x|+t N^{3}\right) u_{N}^{\text {ell, }}(t)\right\|_{L_{x}^{2}} \lesssim\left\|u_{N}(t)\right\|_{\tilde{X}} .
\end{aligned}
$$


Proof. First, we prove (3.12). Set $f:=\mathcal{J}_{ \pm} u_{N}^{\text {hyp }, \pm}$. Note that the support of $f$ is away from the origin. Hence, integration by parts and Plancherel's theorem yield that

$$
\begin{aligned}
\left\|\widetilde{\mathcal{J}}_{ \pm} f(t)\right\|_{L_{x}^{2}}^{2}= & \left\||x|^{\frac{2}{3}} f(t)\right\|_{L_{x}^{2}}^{2}+\left\|t^{t^{\frac{1}{3}}}|x|^{\frac{1}{3}} \partial_{x} f(t)\right\|_{L_{x}^{2}}^{2}+\left\|t^{\frac{2}{3}} \partial_{x}^{2} f(t)\right\|_{L_{x}^{2}}^{2} \\
& \mp 2 t^{\frac{1}{3}} \Im \int_{\mathbb{R}}|x| f(t, x) \overline{\partial_{x} f(t, x)} \mathrm{d} x-2 t^{\frac{2}{3}} \Re \int_{\mathbb{R}}|x|^{\frac{2}{3}} f(t, x) \overline{\partial_{x}^{2} f(t, x)} \mathrm{d} x \\
& \mp 2 t \Im \int_{\mathbb{R}}|x|^{\frac{1}{3}} \partial_{x} f(t, x) \overline{\partial_{x}^{2} f(t, x)} \mathrm{d} x \\
= & \left\||x|^{\frac{2}{3}} f(t)\right\|_{L_{x}^{2}}^{2}+\left\|t^{\frac{1}{3}}|x|^{\frac{1}{3}} \partial_{x} f(t)\right\|_{L_{x}^{2}}^{2}+\left\|t^{\frac{2}{3}} \partial_{x}^{2} f(t)\right\|_{L_{x}^{2}}^{2} \\
& \pm 2 t^{\frac{1}{3}} \int_{\mathbb{R}} \xi\left|\mathcal{F}\left[|\cdot|^{\frac{1}{2}} f\right](t, \xi)\right|^{2} \mathrm{~d} \xi \\
& +2 t^{\frac{2}{3}} \int_{\mathbb{R}}|x|^{\frac{2}{3}}\left|\partial_{x} f(t, x)\right|^{2} \mathrm{~d} x+\frac{4}{9} t^{\frac{2}{3}} \int_{\mathbb{R}}|x|^{-\frac{4}{3}}|f(t, x)|^{2} \mathrm{~d} x \\
& \pm 2 t \int_{\mathbb{R}} \xi\left|\mathcal{F}\left[|\cdot|^{\frac{1}{6}} \partial_{x} f\right](t, \xi)\right|^{2} \mathrm{~d} \xi \\
\geq & \left\||x|^{\frac{2}{3}} f(t)\right\|_{L_{x}^{2}}^{2}+\left\|t^{\frac{1}{3}}|x|^{\frac{1}{3}} \partial_{x} f(t)\right\|_{L_{x}^{2}}^{2}+\left\|t^{\frac{2}{3}} \partial_{x}^{2} f(t)\right\|_{L_{x}^{2}}^{2} \\
& -2 t^{\frac{1}{3}} \int_{\mathbb{R}_{\mp}}|\xi|\left|\mathcal{F}\left[|\cdot|^{\frac{1}{2}} f\right](t, \xi)\right|^{2} \mathrm{~d} \xi \\
& -2 t \int_{\mathbb{R}_{\mp}}|\xi|\left|\mathcal{F}\left[|\cdot|^{\frac{1}{6}} \partial_{x} f\right](t, \xi)\right|^{2} \mathrm{~d} \xi .
\end{aligned}
$$

It follows from (3.11) and (3.8) that

$$
\begin{aligned}
t^{\frac{1}{3}} \int_{\mathbb{R}_{\mp}}|\xi|\left|\mathcal{F}\left[|\cdot|^{\frac{1}{2}} f\right](t, \xi)\right|^{2} \mathrm{~d} \xi & \leq t^{\frac{1}{3}}\left\|P^{\mp}\left|\partial_{x}\right|^{\frac{1}{2}}\left(|\cdot|^{\frac{1}{2}} \mathcal{J}_{ \pm} u_{N}^{\text {hyp, }, \pm}\right)(t)\right\|_{L_{x}^{2}}^{2} \\
& \lesssim N^{-2}\left\|u_{N}(t)\right\|_{L_{x}^{2}}^{2} \\
t \int_{\mathbb{R}_{\mp}}\left|\xi \| \mathcal{F}\left[|\cdot|^{\frac{1}{6}} \partial_{x} f\right](t, \xi)\right|^{2} \mathrm{~d} \xi & \leq t\left\|P^{\mp}\left|\partial_{x}\right|^{\frac{1}{2}}\left(|\cdot|^{\frac{1}{6}} \partial_{x} \mathcal{J}_{ \pm} u_{N}^{\text {hyp }, \pm}\right)(t)\right\|_{L_{x}^{2}}^{2} \\
& \lesssim N^{-2}\left\|u_{N}(t)\right\|_{L_{x}^{2}}^{2} .
\end{aligned}
$$

We therefore obtain

$$
\begin{gathered}
\left\|\tilde{\mathcal{J}}_{ \pm} f(t)\right\|_{L_{x}^{2}}^{2} \geq\left\||x|^{\frac{2}{3}} f(t)\right\|_{L_{x}^{2}}^{2}+\left\|t^{\frac{1}{3}}|x|^{\frac{1}{3}} \partial_{x} f(t)\right\|_{L_{x}^{2}}^{2} \\
+\left\|t^{\frac{2}{3}} \partial_{x}^{2} f(t)\right\|_{L_{x}^{2}}^{2}-C N^{-2}\left\|u_{N}(t)\right\|_{L_{x}^{2}}^{2} .
\end{gathered}
$$

A direct calculation with (3.11) and (1.20) shows that

$$
\begin{aligned}
\widetilde{\mathcal{J}}_{ \pm} f & =\widetilde{\mathcal{J}}_{ \pm} \mathcal{J}_{ \pm} u_{N}^{\text {hyp }, \pm} \\
& = \pm \mathcal{J} u_{N}^{\text {hyp }, \pm}-\frac{i}{3} t^{\frac{1}{3}}|x|^{-\frac{1}{3}} u_{N}^{\text {hyp }, \pm}+\frac{2}{9} t^{\frac{2}{3}}|x|^{-\frac{5}{3}} u_{N}^{\text {hyp }, \pm} \mp \frac{2}{3} t^{\frac{2}{3}}|x|^{-\frac{2}{3}} \partial_{x} u_{N}^{\text {hyp }, \pm} .
\end{aligned}
$$


Moreover, from (1.20) and (3.3), we have

$$
\begin{aligned}
\mathcal{J} u_{N}^{\text {hyp }, \pm}= & \sigma_{N}^{\text {hyp }, \pm} \mathcal{J} u_{N}^{ \pm} \\
& +t\left(\partial_{x}^{3} \sigma_{N}^{\text {hyp }, \pm} \cdot u_{N}^{ \pm}+3 \partial_{x}^{2} \sigma_{N}^{\text {hyp }, \pm} \cdot \partial_{x} u_{N}^{ \pm}+3 \partial_{x} \sigma_{N}^{\text {hyp }, \pm} \cdot \partial_{x}^{2} u_{N}^{ \pm}\right) .
\end{aligned}
$$

Hence, by (3.8), $t N^{4} \geq 1$, and (2.9), we have

$$
\begin{aligned}
\left\|\tilde{\mathcal{J}}_{ \pm} f(t)\right\|_{L_{x}^{2}} & =\left\|\widetilde{\mathcal{J}}_{ \pm} \mathcal{J}_{ \pm} u_{N}^{\text {hyp }, \pm}(t)\right\|_{L_{x}^{2}} \lesssim\left\|\mathcal{J} u_{N}(t)\right\|_{L_{x}^{2}}+N^{-1}\left\|u_{N}(t)\right\|_{L_{x}^{2}} \\
& \lesssim\left\|u_{N}(t)\right\|_{\tilde{X}} .
\end{aligned}
$$

From (3.14), (3.15), and (3.8), we obtain (3.12).

Next, we prove (3.13). We decompose $u_{N}^{\text {ell, }}$ into three parts

$$
\begin{aligned}
u_{N}^{\mathrm{ell}, \pm} & =\sigma_{\leq \frac{2}{\kappa} t N^{3}} u_{N}^{\mathrm{ell}, \pm}+\sigma_{\frac{2}{\kappa} t N^{3}<\cdot<\frac{\kappa}{2} t N^{3}} u_{N}^{\mathrm{ell}, \pm}+\sigma_{\geq \frac{\kappa}{2} t N^{3}} u_{N}^{\mathrm{ell}, \pm} \\
& =: u_{N}^{\mathrm{ell}, \pm, \mathrm{L}}+u_{N}^{\mathrm{ell}, \pm, \mathrm{M}}+u_{N}^{\mathrm{ell}, \pm, \mathrm{H}} .
\end{aligned}
$$

By (1.20), we have

$$
\|x g\|_{L_{x}^{2}}^{2}+\left\|t \partial_{x}^{3} g\right\|_{L_{x}^{2}}^{2}=\|\mathcal{J} g\|_{L_{x}^{2}}^{2}+2 \Im \int_{\mathbb{R}} t x g \cdot \overline{\partial_{x}^{3} g(x)} \mathrm{d} x
$$

for any smooth function $g$.

We consider the estimate of the third part on the right-hand side of (3.16). By the Cauchy-Schwarz inequality, (3.10), (3.16), and (3.4), we have

$$
\begin{aligned}
& \left|\int_{\mathbb{R}} x t u_{N}^{\mathrm{ell}, \pm, \mathrm{H}}(t, x) \cdot \overline{\partial_{x}^{3} u_{N}^{\mathrm{ell}, \pm, \mathrm{H}}(t, x)} \mathrm{d} x\right| \\
& \leq \frac{1}{8}\left\|x u_{N}^{\mathrm{ell}, \pm, \mathrm{H}}(t)\right\|_{L_{x}^{2}}^{2}+2\left\|t \partial_{x}^{3} u_{N}^{\mathrm{ell}, \pm, \mathrm{H}}(t)\right\|_{L_{x}^{2}}^{2} \\
& \leq \frac{1}{8}\left\|x u_{N}^{\mathrm{ell}, \pm, \mathrm{H}}(t)\right\|_{L_{x}^{2}}^{2}+2 \frac{2^{16}}{\kappa^{2}}\left\|P_{\frac{N}{2} \leq \cdot \leq 2 N}\left(x u_{N}^{\mathrm{ell}, \pm, \mathrm{H}}\right)(t)\right\|_{L_{x}^{2}}^{2} \\
& \quad+C t^{2}\left\|\left(1-P_{\frac{N}{2} \leq-\leq 2 N}\right) \partial_{x}^{3}\left(u_{N}^{\mathrm{ell}, \pm, \mathrm{H}}\right)(t)\right\|_{L_{x}^{2}}^{2} \\
& \leq \frac{1}{4}\left\|x u_{N}^{\mathrm{ell}, \pm, \mathrm{H}}(t)\right\|_{L_{x}^{2}}^{2}+C N^{-2}\left\|u_{N}(t)\right\|_{L_{x}^{2}}^{2} .
\end{aligned}
$$

Hence, it follows from taking $g=u_{N}^{\mathrm{ell}, \pm, \mathrm{H}}$ in (3.17) and (3.2) that

$$
\left\|x u_{N}^{\mathrm{ell}, \pm, \mathrm{H}}(t)\right\|_{L_{x}^{2}} \lesssim\left\|u_{N}(t)\right\|_{\tilde{X}}
$$


Next, we consider the estimate of the first part on the right-hand side of (3.16). By the Cauchy-Schwarz inequality, (3.9), and (3.4), we have

$$
\begin{aligned}
& \left|\int_{\mathbb{R}} t x u_{N}^{\mathrm{ell}, \pm, \mathrm{L}}(t, x) \cdot \overline{\partial_{x}^{3} u_{N}^{\mathrm{ell}, \pm, \mathrm{L}}(t, x)} \mathrm{d} x\right| \\
& \quad \leq \frac{1}{8}\left\|t \partial_{x}^{3} u_{N}^{\mathrm{ell}, \pm, \mathrm{L}}(t)\right\|_{L_{x}^{2}}^{2}+2\left\|x u_{N}^{\mathrm{ell}, \pm, \mathrm{L}}(t)\right\|_{L_{x}^{2}}^{2} \\
& \quad \leq \frac{1}{8}\left\|t \partial_{x}^{3} u_{N}^{\mathrm{ell}, \pm, \mathrm{L}}(t)\right\|_{L_{x}^{2}}^{2}+2 \frac{2^{16}}{\kappa^{2}}\left\|t \partial_{x}^{3} P_{\frac{N}{2} \leq \cdot \leq 2 N} u_{N}^{\mathrm{ell}, \pm, \mathrm{L}}(t)\right\|_{L_{x}^{2}}^{2} \\
& \quad+C t^{2} N^{6}\left\|\left(1-P_{\frac{N}{2} \leq \cdot \leq 2 N}\right) u_{N}^{\mathrm{ell}, \pm, \mathrm{L}}(t)\right\|_{L_{x}^{2}}^{2} \\
& \quad \leq \frac{1}{4}\left\|t \partial_{x}^{3} u_{N}^{\mathrm{ell}, \pm, \mathrm{L}}(t)\right\|_{L_{x}^{2}}^{2}+C N^{-2}\left\|u_{N}(t)\right\|_{L_{x}^{2}}^{2} .
\end{aligned}
$$

Hence, it follows from taking $g=u_{N}^{\mathrm{ell}, \pm, \mathrm{L}}$ in (3.17) and (3.2) that

$$
\left\|t \partial_{x}^{3} u_{N}^{\mathrm{ell}, \pm, \mathrm{L}}(t)\right\|_{L_{x}^{2}} \lesssim\left\|u_{N}(t)\right\|_{\tilde{X}}
$$

Finally, we consider the estimate of the second part on the right-hand side of (3.16). It follows from (3.3) to (3.16) that $\operatorname{supp} u_{N}^{\text {ell, }, \mathrm{M}}(t) \subset \mathbb{R}_{\mp}$. In particular, we have $u_{N}^{\mathrm{ell}, \pm, \mathrm{M}}(t, x)=\mathbf{1}_{\mathbb{R}_{\mp}} u_{N}^{\mathrm{ell}, \pm, \mathrm{M}}(t, x)$. By (3.10), we have

$$
\begin{aligned}
\Im & \int_{\mathbb{R}} t x u_{N}^{\mathrm{ell}, \pm, \mathrm{M}}(t, x) \cdot \overline{\partial_{x}^{3} u_{N}^{\mathrm{ell}, \pm, \mathrm{M}}(t, x)} \mathrm{d} x \\
= & \mp t \Im \int_{\mathbb{R}} \sqrt{|x|} u_{N}^{\mathrm{ell}, \pm, \mathrm{M}}(t, x) \overline{\partial_{x}^{3}\left(\sqrt{|x|} u_{N}^{\mathrm{ell}, \pm, \mathrm{M}}(t, x)\right)} \mathrm{d} x \\
& \pm t \Im \int_{\mathbb{R}}|x|^{-1} u_{N}^{\mathrm{ell}, \pm, \mathrm{M}}(t, x) \overline{\partial_{x} u_{N}^{\mathrm{ell}, \pm, \mathrm{M}}(t, x)} \mathrm{d} x \\
& \lesssim t\left\|P^{\mp}\left|\partial_{x}\right|^{\frac{3}{2}}\left(\sqrt{|\cdot|} u_{N}^{\mathrm{ell}, \pm, \mathrm{M}}(t)\right)\right\|_{L_{x}^{2}}^{2}+N^{-2}\left\|u_{N}(t)\right\|_{L_{x}^{2}}^{2} \\
& \lesssim N^{-2}\left\|u_{N}(t)\right\|_{L_{x}^{2}}^{2} .
\end{aligned}
$$

Hence, it follows from (3.17) with $g=u_{N}^{\text {ell, } \pm, \mathrm{M}},(3.2)$, and (3.10) that

$$
t N^{3}\left\|u_{N}^{\mathrm{ell}, \pm, \mathrm{M}}(t)\right\|_{L_{x}^{2}} \lesssim\left\|u_{N}(t)\right\|_{\tilde{X}}
$$

From (3.16), (3.18)-(3.20), (3.9), and (3.10), we obtain (3.13).

By summing up the frequency localized estimates, we obtain the $L^{2}$-estimates. 
Corollary 1. For $t \geq 1$, we have

$$
\begin{aligned}
& \sum_{k=0}^{2} \sum_{\ell=0}^{k}\left\|t^{\frac{k+1}{3}}|x|^{-\frac{4 k+1}{3}+\ell} \partial_{x}^{\ell} u^{\text {hyp }, \pm}(t)\right\|_{L_{x}^{2}} \lesssim\|u(t)\|_{\tilde{X}}, \\
& \sum_{k=0}^{2}\left\|t^{\frac{k}{3}}|x|^{-\frac{k-2}{3}} \mathcal{J}_{ \pm} \partial_{x}^{k} u^{\text {hyp }, \pm}(t)\right\|_{L_{x}^{2}} \lesssim\|u(t)\|_{\tilde{X}}, \\
& \sum_{k=0}^{2}\left\|t^{\frac{k+1}{4}}\left\langle t^{-\frac{1}{4}} x\right\rangle^{-\frac{k}{3}+1} \partial_{x}^{k} u^{\mathrm{ell}}(t)\right\|_{L_{x}^{2}} \lesssim\|u(t)\|_{\widetilde{X}} .
\end{aligned}
$$

The proof is the same as that in Corollary 3.4 in [18]. We thus omit the details here. Moreover, by a repetition of the proof of Proposition 3.5 in [18], we have the pointwise decay estimates.

Proposition 2. For $t \geq 1$ and $k=0,1,2$, we have

$$
\begin{aligned}
& \left|t^{\frac{k+1}{4}}\left\langle t^{-\frac{1}{4}} x\right\rangle^{-\frac{k}{3}+\frac{1}{6}} \partial_{x}^{k} u^{\text {hyp }, \pm}(t, x)\right| \lesssim t^{-\frac{1}{8}}\|u(t)\|_{\widetilde{X}}, \\
& \left|t^{\frac{k+1}{4}}\left\langle t^{-\frac{1}{4}} x\right\rangle^{-\frac{k}{3}+\frac{5}{6}} \partial_{x}^{k} u^{\text {ell }}(t, x)\right| \lesssim t^{-\frac{1}{8}}\|u(t)\|_{\widetilde{X}} .
\end{aligned}
$$

Remark 5. For $t \geq 1$ and $k=0,1,2$, the estimate

$$
\left|t^{\frac{k}{4}+\frac{3}{16}}\left\langle t^{-\frac{1}{4}} x\right\rangle^{-\frac{k}{3}+\frac{1}{3}} \partial_{x}^{k} u^{\text {hyp }, \pm}(t, x)\right| \lesssim\|u(t)\|_{L_{x}^{2}}+t^{-\frac{1}{8}}\|u(t)\|_{\tilde{X}}
$$

holds true. Indeed, by (1.7) and (3.11), we have

$$
\partial_{x}\left(e^{-i \phi} u^{\text {hyp }, \pm}\right)=\mp i t^{-\frac{1}{3}} \mathcal{J}_{ \pm} u^{\text {hyp }, \pm} .
$$

We use the Gagliardo-Nirenberg inequality, (3.26), and (3.8) to obtain

$$
\begin{aligned}
& \left|t^{\frac{k}{4}+\frac{3}{16}}\left\langle t^{-\frac{1}{4}} x\right\rangle^{-\frac{k}{3}+\frac{1}{3}} \partial_{x}^{k} u_{N}^{\text {hyp }, \pm}(t, x)\right| \\
& \quad \lesssim t^{\frac{7}{16}} N^{-k+1}\left\|\partial_{x}^{k} u_{N}^{\text {hyp, }}(t)\right\|_{L_{x}^{\infty}} \\
& \quad \lesssim t^{\frac{13}{48}} N^{-k+1}\left\|\partial_{x}^{k} u_{N}^{\text {hyp, }}(t)\right\|_{L_{x}^{2}}^{\frac{1}{2}}\left\|\mathcal{J}_{ \pm} \partial_{x}^{k} u_{N}^{\text {hyp, }}(t)\right\|_{L_{x}^{2}}^{\frac{1}{2}} \\
& \quad \lesssim t^{-\frac{1}{16}}\left\|u_{N}(t)\right\|_{L_{x}^{2}}^{\frac{1}{2}}\left\|t^{\frac{2}{3}} N^{2} \mathcal{J}_{ \pm} u_{N}^{\text {hyp, },}(t)\right\|_{L_{x}^{2}}^{\frac{1}{2}}+t^{-\frac{1}{8}}\|u(t)\|_{\tilde{X}} \\
& \quad \lesssim\|u(t)\|_{L_{x}^{2}}+t^{-\frac{1}{8}}\|u(t)\|_{\tilde{X}} .
\end{aligned}
$$

Accordingly, from (1.25) and Remark 4, we obtain (1.9) at $t=1$.

\section{Testing by wave packets}

In this section, we prove some properties of wave packets. In Sect. 4.1, we construct wave packets corresponding to the fourth-order Schrödinger equation. Moreover, we show that the wave packet is a good approximate solution to the linear equation. In Sect. 4.2, we prove the output (1.28) is a good approximation of $u$. 
4.1. Construction of wave packets

Let $t \geq 1$. Setting

$$
\lambda:=t^{-\frac{1}{2}} v^{-\frac{1}{3}}=t^{-\frac{1}{4}}\left(t^{\frac{3}{4}} v\right)^{-\frac{1}{3}},
$$

we define, for $|v| \geq t^{-\frac{3}{4}}$,

$$
\Psi_{v}(t, x):=\chi(\lambda(x-v t)) e^{i \phi(t, x)},
$$

where $\chi$ is a smooth function with

$$
\operatorname{supp} \chi \subset\left[-\frac{1}{2}, \frac{1}{2}\right], \quad \int_{\mathbb{R}} \chi(z) \mathrm{d} z=1,
$$

and $\phi$ is defined by (1.7). The spatial support of $\Psi_{v}$ is included in $\left[\frac{v t}{2}, \frac{3}{2} v t\right]$ for $v>0$ or in $\left[\frac{3}{2} v t, \frac{v t}{2}\right]$ for $v<0$. In particular, the sign of $x$ is the same as that of $v$.

We show that $\Psi_{v}(t, x)$ is essentially localized at frequency

$$
\xi_{v}:=v^{\frac{1}{3}}=t^{-\frac{1}{4}}\left(t^{\frac{3}{4}} v\right)^{\frac{1}{3}}
$$

in the following sense (see Lemma 4.1 in [18], for example):

Lemma 5. For $t \geq 1$ and $|v| \geq t^{-\frac{3}{4}}$, we have

$$
\mathcal{F}\left[\Psi_{v}\right](t, \xi)=\frac{1}{\sqrt{3}} \lambda^{-1} \chi_{1}\left(\lambda^{-1}\left(\xi-\xi_{v}\right), \lambda^{-1} \xi_{v}\right) e^{-\frac{1}{4} i t \xi^{4}},
$$

where $\chi_{1}(\cdot, \alpha) \in \mathcal{S}(\mathbb{R})$ satisfies

$$
\sup _{|\alpha| \geq 1} \sup _{\zeta \in \mathbb{R}}\left|\langle\zeta\rangle^{k} \partial_{\zeta}^{\ell} \chi_{1}(\zeta, \alpha)\right| \lesssim_{k, \ell} 1
$$

for any $k, \ell \in \mathbb{N}_{0}$. Moreover, there exists a constant $C_{1}>0$ such that for any $|\alpha| \geq 1$,

$$
\left|\int_{\mathbb{R}} \chi_{1}(\zeta, \alpha) \mathrm{d} \zeta-1\right| \leq \frac{C_{1}}{|\alpha|} .
$$

For $|v| \geq t^{-\frac{3}{4}}$, we define the nearest dyadic number to $\left|\xi_{v}\right|$ by $N_{v} \in 2^{\mathbb{Z}}$. Then, we have

$$
\frac{3}{4} N_{v} \leq\left|\xi_{v}\right| \leq \frac{3}{2} N_{v}
$$

Moreover, let \pm be the sign of $v$ :

$$
\pm v=|v|
$$

Lemma 5 yields the following bound.

Lemma 6. For $|v| \geq t^{-\frac{3}{4}}, a \geq 0$, and $k \in \mathbb{N}_{0}$, we have

$$
\left\|\left(1-P_{N_{v}}^{ \pm}\right) \partial_{x}^{k} \Psi_{v}(t)\right\|_{L_{x}^{1}} \lesssim a, k t^{\frac{1-k}{4}}\left(t^{\frac{3}{4}}|v|\right)^{-a},
$$

where \pm is as in (4.8). 
Proof. It suffices to show that

$$
\left|\left(1-P_{N_{v}}^{ \pm}\right) \partial_{x}^{k} \Psi_{v}(t, x)\right| \lesssim a, k t^{-\frac{k}{4}}\left(t^{\frac{3}{4}}|v|\right)^{-a} \min \left(1,|x|^{-1} t^{\frac{1}{4}}\right)^{2}
$$

for any $k \in \mathbb{N}_{0}$ and $a \geq 0$. Indeed, once we have (4.9), we obtain

$$
\begin{aligned}
\left\|\left(1-P_{N_{v}}^{ \pm}\right) \partial_{x}^{k} \Psi_{v}(t)\right\|_{L_{x}^{1}} \leq & \left\|\left(1-P_{N_{v}}^{ \pm}\right) \partial_{x}^{k} \Psi_{v}(t)\right\|_{L_{x}^{1}\left(\left\{|x| \leq t^{\frac{1}{4}}\right\}\right)} \\
& +\left\|\left(1-P_{N_{v}}^{ \pm}\right) \partial_{x}^{k} \Psi_{v}(t)\right\|_{L_{x}^{1}\left(\left\{|x| \geq t^{\frac{1}{4}}\right\}\right)} \\
& \lesssim t^{\frac{1-k}{4}}\left(t^{\frac{3}{4}}|v|\right)^{-a} .
\end{aligned}
$$

In what follows, we show (4.9). By Lemma 5 and changing variable $\zeta=\lambda^{-1}\left(\xi-\xi_{v}\right)$, we have

$$
\begin{aligned}
\left|\left(1-P_{N_{v}}^{ \pm}\right) \partial_{x}^{k} \Psi_{v}(t, x)\right|= & \mid \frac{1}{\sqrt{6 \pi}} \int_{\mathbb{R}} e^{i x\left(\lambda \zeta+\xi_{v}\right)}\left(1-\sigma_{N_{v}}^{ \pm}\left(\lambda \zeta+\xi_{v}\right)\right) \\
& \times\left(\lambda \zeta+\xi_{v}\right)^{k} \chi_{1}\left(\zeta, \lambda^{-1} \xi_{v}\right) e^{-\frac{i}{4} t\left(\lambda \zeta+\xi_{v}\right)^{4}} \mathrm{~d} \zeta \mid
\end{aligned}
$$

Here, we note that

$$
\begin{aligned}
& \operatorname{supp}\left(1-\sigma_{N_{v}}^{ \pm}\left(\lambda \zeta+\xi_{v}\right)\right) \\
& \quad \subset\left\{\left|\lambda \zeta+\xi_{v}\right| \leq \frac{N_{v}}{2}\right\} \cup\left\{\frac{N_{v}}{2} \leq \mp\left(\lambda \zeta+\xi_{v}\right) \leq 2 N_{v}\right\} \cup\left\{\left|\lambda \zeta+\xi_{v}\right| \geq 2 N_{v}\right\} \\
& \quad=: I_{1} \cup I_{2} \cup I_{3} .
\end{aligned}
$$

Then, we have

$$
|\zeta| \gtrsim\left(t^{\frac{3}{4}}|v|\right)^{\frac{2}{3}}
$$

for $\zeta \in I_{1} \cup I_{2} \cup I_{3}$. In fact, on $I_{1}$, it follows from the triangle inequality, (4.7), (4.1), and (4.4) that

$$
|\zeta| \geq \lambda^{-1}\left(\left|\xi_{v}\right|-\frac{N_{v}}{2}\right) \geq \lambda^{-1} \frac{N_{v}}{4} \sim\left(t^{\frac{3}{4}}|v|\right)^{\frac{2}{3}} .
$$

Similarly, on $I_{3}$, it follows from the triangle inequality, (4.7), (4.1), and (4.4) that

$$
|\zeta| \geq \lambda^{-1}\left(2 N_{v}-\left|\xi_{v}\right|\right) \geq \lambda^{-1} \frac{N_{v}}{2} \sim\left(t^{\frac{3}{4}}|v|\right)^{\frac{2}{3}}
$$

Moreover, by (4.8), we have $\mp\left(\lambda \zeta+\xi_{v}\right)=-|\lambda| \zeta-\left|\xi_{v}\right|=|\lambda \zeta|-\left|\xi_{v}\right|$ on $I_{2}$. Hence, (4.1) and (4.4) yield that

$$
|\zeta| \geq|\lambda|^{-1}\left(\left|\xi_{v}\right|+\frac{N_{v}}{2}\right) \sim\left(t^{\frac{3}{4}}|v|\right)^{\frac{2}{3}}
$$

Therefore, (4.11) holds. 
It follows from (4.10), (4.11), and (4.5) that

$$
\begin{aligned}
\left|\left(1-P_{N_{v}}^{ \pm}\right) \partial_{x}^{k} \Psi_{v}(t, x)\right| & \lesssim\left(t^{\frac{3}{4}}|v|\right)^{-\frac{2}{3} a^{\prime}} \int_{\mathbb{R}}\left|\lambda \zeta+\xi_{v}\right|^{k}\langle\zeta\rangle^{a^{\prime}}\left|\chi_{1}\left(\zeta, \lambda^{-1} \xi_{v}\right)\right| \mathrm{d} \zeta \\
& \lesssim t^{-\frac{k}{4}}\left(t^{\frac{3}{4}}|v|\right)^{-\frac{2}{3} a^{\prime}+\frac{k}{3}}
\end{aligned}
$$

for any $a^{\prime}>0$. Hence, by (4.12) and choosing $a^{\prime}>\frac{3}{2} a+\frac{k}{2}$, we obtain (4.9) for $|x| \leq t^{\frac{1}{4}}$. Moreover, we use integration by parts twice to (4.10), (4.11), (4.5), (4.1), and (4.4) to have

$$
\begin{aligned}
\left|\left(1-P_{N_{v}}^{ \pm}\right) \partial_{x}^{k} \Psi_{v}(t, x)\right| & \lesssim\left(t^{\frac{3}{4}}|v|\right)^{-\frac{2}{3} a^{\prime}+\frac{k}{3}} \cdot|x \lambda|^{-2}\left(\left|\lambda^{2} \xi_{v}^{k-2}\right|+\left|\xi_{v}^{k} t^{2} \lambda^{2} \xi_{v}^{6}\right|\right) \\
& \lesssim t^{-\frac{k}{4}}\left(t^{\frac{3}{4}}|v|\right)^{-\frac{2}{3} a^{\prime}+\frac{k}{3}+2} \cdot\left(|x|^{-1} t^{\frac{1}{4}}\right)^{2}
\end{aligned}
$$

for any $a^{\prime}>0$. Hence, (4.9) for $|x| \geq t^{\frac{1}{4}}$ follows from choosing $a^{\prime}>\frac{2}{3} a+\frac{k}{2}+3$ in (4.13). We therefore obtain (4.9), which concludes the proof.

Next, we show that $\Psi_{v}$ is a good approximate solution for the linear equation. For $|v| \geq t^{-\frac{3}{4}}$, a direct calculation with (4.2) and (4.1) shows that

$$
\partial_{t} \Psi_{v}(t, x)=-\frac{x+v t}{2 t} \lambda \chi^{\prime}(\lambda(x-v t)) e^{i \phi(t, x)}+i \partial_{t} \phi(t, x) \chi(\lambda(x-v t)) e^{i \phi(t, x)} .
$$

By (1.7), we have

$$
-\partial_{t} \phi=\frac{1}{4}\left(\partial_{x} \phi\right)^{4}
$$

It follows from (1.19), (4.14), and (4.15) that

$$
\left(\mathcal{L} \Psi_{v}\right)(t, x)=i \frac{e^{i \phi(t, x)}}{t \lambda} \partial_{x}(\tilde{\chi}(t, x))+O\left(t^{-1}\left(t^{\frac{3}{4}}|v|\right)^{-\frac{4}{3}} \chi(\lambda(x-t v))\right),
$$

where

$$
\begin{aligned}
\tilde{\chi}(t, x) & :=\lambda \frac{x-v t}{2} \chi(\lambda(x-v t)) \\
& -i \frac{3}{2} \lambda^{2} t^{\frac{1}{3}} x^{\frac{2}{3}} \chi^{\prime}(\lambda(x-v t))-\lambda^{3} t^{\frac{2}{3}} x^{\frac{1}{3}} \chi^{\prime \prime}(\lambda(x-v t))
\end{aligned}
$$

has the same localization of $\chi(\lambda(x-v t))$. More precisely, by (4.1), and (4.4), we can write $\tilde{\chi}$ as follows:

$$
\begin{aligned}
\tilde{\chi}(t, x)= & \lambda \frac{x-v t}{2} \chi(\lambda(x-v t))-i \frac{3}{2} \lambda^{\frac{4}{3}} t^{\frac{1}{3}}(\lambda(x-v t)+\lambda v t)^{\frac{2}{3}} \chi^{\prime}(\lambda(x-v t)) \\
& -\lambda^{\frac{8}{3}} t^{\frac{2}{3}}(\lambda(x-v t)+\lambda v t)^{\frac{1}{3}} \chi^{\prime \prime}(\lambda(x-v t)) \\
= & \tilde{\chi}_{0}\left(\lambda(x-v t), \lambda^{-1} \xi_{v}\right),
\end{aligned}
$$

where

$$
\tilde{\chi}_{0}(z, \alpha):=\frac{z}{2} \chi(z)-i \frac{3}{2} \alpha^{-\frac{2}{3}}(z+\alpha)^{\frac{2}{3}} \chi^{\prime}(z)-\alpha^{-\frac{4}{3}}(z+\alpha)^{\frac{1}{3}} \chi^{\prime \prime}(z) .
$$




\subsection{Approximation of $u$}

In this subsection, by using wave packets constructed in Sect. 4.1 , we prove the output $\gamma(t, v)$ defined in (1.28) is a "good" approximation of $u$.

Let $C_{2}>0$ be the constant appearing in (4.5) with $k=2$ and $\ell=0$, that is,

$$
\sup _{|\alpha| \geq 1} \sup _{\zeta \in \mathbb{R}}\left|\langle\zeta\rangle^{2} \chi_{1}(\zeta, \alpha)\right| \leq C_{2}
$$

For $t \geq 1$, we define

$$
\Omega(t):=\left\{v \in \mathbb{R}:|v| \geq C_{*} t^{-\frac{3}{4}}\right\}
$$

where

$$
C_{*}:=\left(2\left(C_{1}+C_{2}+1\right)\right)^{\frac{3}{2}} .
$$

Here, $C_{1}$ is the constant appearing in (4.6). The large constant $C_{*}$ is needed to show the pointwise estimate (4.24) in the frequency space below.

The main goal in this subsection is to prove the following proposition:

Proposition 3. For $t \geq 1$ and $k=0,1,2$, we have the bound

$$
\partial_{x}^{k} u(t, v t)=i^{k} \lambda v^{\frac{k}{3}} e^{i \phi(t, v t)} \gamma(t, v)+R_{k}(t, v),
$$

where $\gamma$ and $\phi$ are defined in (1.28) and (1.7), respectively, and $R_{k}$ is a function satisfying

$$
\begin{aligned}
& \left\|t^{\frac{k+1}{4}}\left(t^{\frac{3}{4}}|v|\right)^{-\frac{k}{3}+\frac{1}{2}} R_{k}(t, v)\right\|_{L_{v}^{\infty}(\Omega(t))}+\left\|t^{\frac{k}{4}+\frac{5}{8}}\left(t^{\frac{3}{4}}|v|\right)^{-\frac{k}{3}+\frac{1}{3}} R_{k}(t, v)\right\|_{L_{v}^{2}(\Omega(t))} \\
& \quad \lesssim t^{-\frac{1}{8}}\|u(t)\|_{\tilde{X}} .
\end{aligned}
$$

Moreover, in the frequency space, we have

$$
\widehat{u}\left(t, \xi_{v}\right)=\sqrt{3} e^{-\frac{1}{4} i t \xi_{v}^{4}} \gamma(t, v)+R_{\xi}(t, v),
$$

where $R_{\xi}$ is a function satisfying

$$
\left\|\left(t^{\frac{3}{4}}|v|\right)^{\frac{1}{6}} R_{\xi}(t, v)\right\|_{L_{v}^{\infty}(\Omega(t))}+\left\|t^{\frac{3}{8}} R_{\xi}(t, v)\right\|_{L_{v}^{2}(\Omega(t))} \lesssim t^{-\frac{1}{8}}\|u(t)\|_{\tilde{X}} .
$$

Before the proof of Proposition 3, we provide two preliminary lemmas.

Lemma 7. For $t \geq 1$, we have

$$
\left\|v^{-\frac{1}{3}} \int_{\mathbb{R}}|f(t, x) \chi(\lambda(x-v t))| \mathrm{d} x\right\|_{L_{v}^{2}(\Omega(t))} \lesssim\|f(t, \cdot)\|_{L_{x}^{2}\left(|x| \geq t^{\frac{1}{4}}\right)} .
$$

Proof. By a change of variables using $z=\lambda(x-v t)$ and (4.1),

$$
\text { L.H.S. of }(4.25)=t^{\frac{1}{2}}\left\|\int_{\mathbb{R}}\left|f\left(t, t^{\frac{1}{2}} v^{\frac{1}{3}} z+v t\right) \chi(z)\right| \mathrm{d} z\right\|_{L_{v}^{2}(\Omega(t))} .
$$


Setting $\widetilde{v}=t^{\frac{1}{2}} v^{\frac{1}{3}} z+v t$, we note that

$$
\begin{gathered}
\left|t^{-\frac{1}{4}} \widetilde{v}\right|=t^{\frac{3}{4}}|v|\left|1+\left(t^{\frac{3}{4}} v\right)^{-\frac{2}{3}} z\right| \geq 1, \\
\left|\frac{\mathrm{d} \widetilde{v}}{\mathrm{~d} v}\right|=t\left|1+\frac{1}{3}\left(t^{\frac{3}{4}} v\right)^{-\frac{2}{3}} z\right| \geq \frac{t}{2}
\end{gathered}
$$

for $v \in \Omega(t)$ and $|z| \leq \frac{1}{2}$. Then, we have

$$
\begin{aligned}
\text { L.H.S. of (4.25) } & \lesssim t^{\frac{1}{2}} \int_{\mathbb{R}}\left\|f\left(t, t^{\frac{1}{2}} v^{\frac{1}{3}} z+v t\right)\right\|_{L_{v}^{2}(\Omega(t))}|\chi(z)| \mathrm{d} z \\
& \lesssim\|f(t, \cdot)\|_{L_{x}^{2}\left(|x| \geq t^{\frac{1}{4}}\right)}
\end{aligned}
$$

which shows (4.25).

The second lemma says that we can replace $\left(i \xi_{v}\right)^{k} u$ in (1.28) with $\partial_{x}^{k} u^{\text {hyp, }}$.

Lemma 8. For $t \geq 1$ and $k=0,1,2$, we have

$$
i^{k} \lambda v^{\frac{k}{3}} \gamma(t, v)=\lambda \int_{\mathbb{R}} \partial_{x}^{k} u^{\text {hyp }, \pm}(t, x) \overline{\Psi_{v}(t, x)} \mathrm{d} x+R_{k}(t, v),
$$

where $\pm i$ is as in (4.8) and $R_{k}$ is a function satisfying (4.23).

Proof. First, we note that

$$
i^{k} \lambda v^{\frac{k}{3}} \gamma(t, v)=i^{k} \lambda v^{\frac{k}{3}} \int_{\mathbb{R}} u^{\text {hyp }, \pm}(t, x) \overline{\Psi_{v}(t, x)} \mathrm{d} x+R_{k}(t, v) .
$$

Indeed, it follows from (1.28), (3.6), (3.5) and supp $\Psi_{v}(t) \subset \mathbb{R}_{ \pm}$that

$$
\gamma(t, v)=\int_{\mathbb{R}} u^{\mathrm{hyp}, \pm}(t, x) \overline{\Psi_{v}(t, x)} \mathrm{d} x+\int_{\mathbb{R}} u^{\mathrm{ell}}(t, x) \overline{\Psi_{v}(t, x)} \mathrm{d} x .
$$

For the second part on the right-hand side of (4.28), we use (4.2), (4.1), and (3.25) to obtain that

$$
\begin{aligned}
\left|\int_{\mathbb{R}} u^{\mathrm{ell}}(t, x) \overline{\Psi_{v}(t, x)} \mathrm{d} x\right| & \lesssim|\lambda|^{-1}\left(t^{\frac{3}{4}}|v|\right)^{-\frac{5}{6}}\left\|\left(t^{-\frac{1}{4}}|x|\right)^{\frac{5}{6}} u^{\mathrm{ell}}(t)\right\|_{L_{x}^{\infty}} \\
& \lesssim\left(t^{\frac{3}{4}}|v|\right)^{-\frac{1}{2}} \cdot t^{-\frac{1}{8}}\|u(t)\|_{\tilde{X}} .
\end{aligned}
$$

Moreover, it follows from Lemma 7 and (3.23) that

$$
\left\|\int_{\mathbb{R}} u^{\mathrm{ell}}(t, x) \overline{\Psi_{v}(t, x)} \mathrm{d} x\right\|_{L_{v}^{2}(\Omega(t))} \lesssim t^{-\frac{1}{4}}\left\|\left\langle t^{-\frac{1}{4}} x\right\rangle^{\frac{1}{3}} u^{\mathrm{ell}}(t)\right\|_{L_{x}^{2}} \lesssim t^{-\frac{3}{8}} \cdot t^{-\frac{1}{8}}\|u(t)\|_{\tilde{X}}
$$

Since (4.1) yields $\lambda|v|^{\frac{k}{3}}=t^{-\frac{k+1}{4}}\left(t^{\frac{3}{4}}|v|\right)^{\frac{k}{3}-\frac{1}{3}}$, (4.27) follows from (4.28) and (4.30). 
Second, we prove (4.26). Since (4.26) with $k=0$ is (4.27) with $k=0$, we only consider the case $k=1,2$. A direct calculation with (1.7) and (4.2) shows that

$$
\begin{aligned}
u^{\text {hyp }, \pm}(t, x) \overline{\Psi_{v}(t, x)}= & -i v^{-\frac{1}{3}} \partial_{x} u^{\text {hyp }, \pm}(t, x) \overline{\Psi_{v}(t, x)} \\
& -i t^{\frac{1}{3}}\left(x^{-\frac{1}{3}}-(v t)^{-\frac{1}{3}}\right) \partial_{x} u^{\text {hyp }, \pm}(t, x) \overline{\Psi_{v}(t, v)} \\
& +i t^{\frac{1}{3}} x^{-\frac{1}{3}} \partial_{x}\left(e^{-i \phi} u^{\text {hyp }, \pm}\right)(t, x) \chi(\lambda(x-v t)) .
\end{aligned}
$$

Here, (4.1), (4.2), and (3.24) yield that

$$
\begin{aligned}
& |v|^{-\frac{k-1}{3}}\left|\int_{\mathbb{R}} t^{\frac{1}{3}}\left(x^{-\frac{1}{3}}-(v t)^{-\frac{1}{3}}\right) \partial_{x}^{k} u^{\mathrm{hyp}, \pm}(t, x) \overline{\Psi_{v}(t, x)} \mathrm{d} x\right| \\
& \quad \lesssim t^{\frac{k}{4}}\left(t^{\frac{3}{4}}|v|\right)^{-\frac{5}{6}}|\lambda|^{-1}\left\|\left\langle t^{-\frac{1}{4}} x\right\rangle^{-\frac{k}{3}+\frac{1}{6}} \partial_{x}^{k} u^{\mathrm{hyp}, \pm}(t, x)\right\|_{L_{x}^{\infty}} \\
& \quad \lesssim\left(t^{\frac{3}{4}}|v|\right)^{-\frac{1}{2}} \cdot t^{-\frac{1}{8}}\|u(t)\|_{\tilde{X}}
\end{aligned}
$$

for $k=1,2$. By Lemma 7 and (3.21), we have

$$
\begin{aligned}
& \left\|v^{-\frac{k-1}{3}} \int_{\mathbb{R}} t^{\frac{1}{3}}\left(x^{-\frac{1}{3}}-(v t)^{-\frac{1}{3}}\right) \partial_{x}^{k} u^{\text {hyp }, \pm}(t, x) \overline{\Psi_{v}(t, x)} \mathrm{d} x\right\|_{L_{v}^{2}(\Omega(t))} \\
& \lesssim t^{-\frac{1}{2}}\left\|\left(\frac{x}{t}\right)^{-\frac{k+1}{3}} \partial_{x}^{k} u^{\mathrm{hyp}, \pm}(t)\right\|_{L_{x}^{2}} \\
& \lesssim t^{-\frac{3}{8}} \cdot t^{-\frac{1}{8}}\|u(t)\|_{\tilde{X}} .
\end{aligned}
$$

Moreover, Hölder's inequality, (3.26), (3.22), and (4.1) imply that

$$
\begin{aligned}
& \left|v^{-\frac{k-1}{3}} \int_{\mathbb{R}} t^{\frac{1}{3}} x^{-\frac{1}{3}} \partial_{x}\left(e^{-i \phi} \partial_{x}^{k-1} u^{\mathrm{hyp}, \pm}\right)(t, x) \chi(\lambda(x-v t)) \mathrm{d} x\right| \\
& \quad \lesssim t^{\frac{k}{3}-\frac{7}{12}}\left(t^{\frac{3}{4}}|v|\right)^{-1}|\lambda|^{-\frac{1}{2}}\left\||x|^{-\frac{k-3}{3}} \mathcal{J}_{ \pm} \partial_{x}^{k-1} u^{\mathrm{hyp}, \pm}(t)\right\|_{L_{x}^{2}} \\
& \quad \lesssim\left(t^{\frac{3}{4}}|v|\right)^{-\frac{5}{6}} \cdot t^{-\frac{1}{8}}\|u(t)\|_{\tilde{X}}
\end{aligned}
$$

for $k=1,2$. In addition, (4.34) yields that

$$
\begin{aligned}
& \left\|v^{-\frac{k-1}{3}} \int_{\mathbb{R}} t^{\frac{1}{3}} x^{-\frac{1}{3}} \partial_{x}\left(e^{-i \phi} \partial_{x}^{k-1} u^{\mathrm{hyp}, \pm}\right)(t, x) \chi(\lambda(x-v t)) \mathrm{d} x\right\|_{L_{v}^{2}(\Omega(t))} \\
& \quad \lesssim\left\|\left(t^{\frac{3}{4}} v\right)^{-\frac{5}{6}}\right\|_{L_{v}^{2}(\Omega(t))} \cdot t^{-\frac{1}{8}}\|u(t)\|_{\tilde{X}} \\
& \quad \lesssim t^{-\frac{3}{8}} \cdot t^{-\frac{1}{8}}\|u(t)\|_{\tilde{X}} .
\end{aligned}
$$

Therefore, by (4.27) and (4.31)-(4.35), we obtain (4.26).

We are now in position to prove Proposition 3. 
Proof of Proposition 3. First, we show (4.22). Let \pm be as in (4.8). Then, it follows from (3.5) that $u^{\text {hyp }}(t, v t)=u^{\text {hyp, }{ }^{ \pm}}(t, v t)$. By (3.6), (3.23), and (3.25), we have

$$
\partial_{x}^{k} u(t, v t)=\partial_{x}^{k} u^{\mathrm{hyp}, \pm}(t, v t)+R_{k}(t, v),
$$

where $R_{k}$ satisfies (4.23). We set

$$
w_{k}(t, x):=e^{-i \phi(t, x)} \partial_{x}^{k} u^{\text {hyp }, \pm}(t, x) .
$$

By (4.36), Lemma 8, and (4.3), we have

$$
\begin{aligned}
& \partial_{x}^{k} u(t, v t)-i^{k} \lambda v^{\frac{k}{3}} e^{i \phi(t, v t)} \gamma(t, v) \\
& \quad=\lambda e^{i \phi(t, v t)} \int_{\mathbb{R}}\left(w_{k}(t, v t)-w_{k}(t, x)\right) \chi(\lambda(x-v t)) \mathrm{d} x+R_{k}(t, v) .
\end{aligned}
$$

It follows from (4.37) and (3.26) that

$$
\partial_{x} w_{k}(t, x)=\mp i t^{-\frac{1}{3}} \mathcal{J}_{ \pm} \partial_{x}^{k} u^{\text {hyp }, \pm} .
$$

With a change of variables using $z=\lambda(x-v t)$, the mean value theorem, (4.39), Hölder's inequality in $\theta,(3.22)$, and (4.1), we see that

$$
\begin{aligned}
& |\lambda| \int_{\mathbb{R}}\left|\left(w_{k}(t, v t)-w_{k}(t, x)\right) \chi(\lambda(x-v t))\right| \mathrm{d} x \\
& \quad \leq|\lambda|^{-1} \int_{\mathbb{R}}\left|\int_{0}^{1} \partial_{x} w_{k}\left(t, v t+(1-\theta) \lambda^{-1} z\right) \mathrm{d} \theta \cdot z \chi(z)\right| \mathrm{d} z \\
& \quad \lesssim t^{-\frac{k+1}{4}}\left(t^{\frac{3}{4}}|v|\right)^{\frac{k}{3}-\frac{1}{2}} \cdot t^{-\frac{1}{8}}\|u(t)\|_{\tilde{X}} .
\end{aligned}
$$

From (4.38) and (4.40), we obtain the $L^{\infty}$-estimate in (4.22).

Moreover, a change of variables using $z=\lambda(x-v t)$ and $\tilde{v}=v t+(1-\theta) \lambda^{-1} z$, and (3.22) give

$$
\begin{aligned}
& \left\|t^{\frac{k}{4}+\frac{5}{8}}\left(t^{\frac{3}{4}} v\right)^{-\frac{k}{3}+\frac{1}{3}} \lambda \int_{\mathbb{R}}\left|w_{k}(t, v t)-w_{k}(t, x)\right| \chi(\lambda(x-v t)) \mathrm{d} x\right\|_{L_{v}^{2}(\Omega(t))} \\
& \quad \leq t^{\frac{k}{3}-\frac{1}{8}}\left\|\widetilde{v}^{-\frac{k-2}{3}}\left(\mathcal{J}_{ \pm} \partial_{x}^{k} u^{\mathrm{hyp}, \pm}\right)(t, \widetilde{v})\right\|_{L_{\widetilde{v}}^{2}} \\
& \quad \lesssim t^{-\frac{1}{8}}\|u(t)\| \widetilde{X} .
\end{aligned}
$$

Hence, the $L^{2}$-estimate in (4.22) follows from (4.38) and (4.41).

Next, we consider the estimates in the frequency spaces. By (1.28), Lemmas 5 and 6, and Proposition 2, we have

$$
\begin{aligned}
\sqrt{3} e^{-\frac{1}{4} i t \xi_{v}^{4}} \gamma(t, v)= & e^{-\frac{1}{4} i t \xi_{v}^{4}} \int_{\mathbb{R}_{ \pm}} \widehat{u}(t, \xi) \lambda^{-1} \overline{\chi_{1}\left(\lambda^{-1}\left(\xi-\xi_{v}\right), \lambda^{-1} \xi_{v}\right) e^{-\frac{1}{4} i t \xi^{4}}} \mathrm{~d} \xi \\
& +O\left(\left(t^{\frac{3}{4}}|v|\right)^{-1} \cdot t^{-\frac{1}{8}}\|u(t)\| \tilde{X}\right) .
\end{aligned}
$$


By changing variable $\zeta=\lambda^{-1}\left(\xi-\xi_{v}\right),(4.19),(4.1)$, and (4.7), we have

$$
\begin{aligned}
\left|\int_{\mathbb{R}_{\mp}} \lambda^{-1} \chi_{1}\left(\lambda^{-1}\left(\xi-\xi_{v}\right), \lambda^{-1} \xi_{v}\right) \mathrm{d} \xi\right| & =\left|\int_{-\infty}^{-\lambda^{-1} \xi_{v}} \chi_{1}\left(\zeta, \lambda^{-1} \xi_{v}\right) \mathrm{d} \zeta\right| \\
& \leq C_{2} \int_{-\infty}^{-\lambda^{-1} \xi_{v}}\langle\zeta\rangle^{-2} d \zeta \leq C_{2}\left(t^{\frac{3}{4}}|v|\right)^{-\frac{2}{3}}
\end{aligned}
$$

It follows from (4.6) and (4.43) that

$$
\begin{aligned}
\mid 1 & -\int_{\mathbb{R}_{ \pm}} \lambda^{-1} \chi_{1}\left(\lambda^{-1}\left(\xi-\xi_{v}\right), \lambda^{-1} \xi_{v}\right) \mathrm{d} \xi \mid \\
& \leq C_{1}\left(t^{\frac{3}{2}}|v|\right)^{-\frac{2}{3}}+\left|\int_{\mathbb{R}_{\mp}} \lambda^{-1} \chi_{1}\left(\lambda^{-1}\left(\xi-\xi_{v}\right), \lambda^{-1} \xi_{v}\right) \mathrm{d} \xi\right| \\
& \leq\left(C_{1}+C_{2}\right)\left(t^{\frac{3}{4}}|v|\right)^{-\frac{2}{3}} .
\end{aligned}
$$

Hence, it follows from (4.42) and (4.44) that

$$
\begin{aligned}
& \mid \widehat{u}(t,\left.\xi_{v}\right)-\sqrt{3} e^{-\frac{1}{4} i t \xi_{v}^{4}} \gamma(t, v) \mid \\
& \leq \mid \int_{\mathbb{R}_{ \pm}}\left(\widehat{u}\left(t, \xi_{v}\right) e^{\frac{1}{4} i t \xi_{v}^{4}}-\widehat{u}(t, \xi) e^{\frac{1}{4} i t \xi^{4}}\right) \lambda^{-1} \overline{\chi_{1}\left(\lambda^{-1}\left(\xi-\xi_{v}\right), \lambda^{-1} \xi_{v}\right)} \mathrm{d} \xi \\
& \quad+\left(C_{1}+C_{2}\right)\left(t^{\frac{3}{4}}|v|\right)^{-\frac{2}{3}}\left|\widehat{u}\left(t, \xi_{v}\right)\right|+C\left(t^{\frac{3}{4}}|v|\right)^{-1} \cdot t^{-\frac{1}{8}}\|u(t)\|_{\tilde{X}} .
\end{aligned}
$$

By (1.28), Proposition 2, (4.2), and (4.1), we have

$$
|\gamma(t, v)| \lesssim\left(t^{\frac{3}{4}}|v|\right)^{-\frac{1}{6}}|\lambda|^{-1}\left\|\left\langle t^{-\frac{1}{4}} x\right\rangle^{\frac{1}{6}} u(t)\right\|_{L_{x}^{\infty}} \lesssim\left(t^{\frac{3}{4}}|v|\right)^{\frac{1}{6}} \cdot t^{-\frac{1}{8}}\|u(t)\|_{\tilde{X}} .
$$

It follows from (4.46) and (4.21) that

$$
\begin{aligned}
\left(C_{1}\right. & \left.+C_{2}\right)\left(t^{\frac{3}{4}}|v|\right)^{-\frac{2}{3}}\left|\widehat{u}\left(t, \xi_{v}\right)\right| \\
\leq & \left(C_{1}+C_{2}\right)\left(t^{\frac{3}{4}}|v|\right)^{-\frac{2}{3}}\left|\widehat{u}\left(t, \xi_{v}\right)-\sqrt{3} e^{-\frac{1}{4} i t \xi_{v}^{4}} \gamma(t, v)\right| \\
& +\sqrt{3}\left(C_{1}+C_{2}\right)\left(t^{\frac{3}{4}}|v|\right)^{-\frac{2}{3}}|\gamma(t, v)| \\
\leq & \frac{1}{2}\left|\widehat{u}\left(t, \xi_{v}\right)-\sqrt{3} e^{-\frac{1}{4} i t \xi_{v}^{4}} \gamma(t, v)\right|+C\left(t^{\frac{3}{4}}|v|\right)^{-\frac{1}{2}} \cdot t^{-\frac{1}{8}}\|u(t)\|_{\tilde{X}}
\end{aligned}
$$

for $v \in \Omega(t)$. Therefore, (4.45) and (4.47) yield that

$$
\begin{aligned}
& \left|\widehat{u}\left(t, \xi_{v}\right)-\sqrt{3} e^{-\frac{1}{4} i t \xi_{v}^{4}} \gamma(t, v)\right| \\
& \lesssim\left|\int_{\mathbb{R}_{ \pm}}\left(\widehat{u}\left(t, \xi_{v}\right) e^{\frac{1}{4} i t \xi_{v}^{4}}-\widehat{u}(t, \xi) e^{\frac{1}{4} i t \xi^{4}}\right) \lambda^{-1} \overline{\chi_{1}\left(\lambda^{-1}\left(\xi-\xi_{v}\right), \lambda^{-1} \xi_{v}\right)} \mathrm{d} \xi\right| \\
& +\left(t^{\frac{3}{4}}|v|\right)^{-\frac{1}{2}} \cdot t^{-\frac{1}{8}}\|u(t)\|_{\tilde{X}} .
\end{aligned}
$$


With the mean value theorem and a change of variables using $\zeta=\lambda^{-1}\left(\xi-\xi_{v}\right)$, we have

$$
\begin{aligned}
& \left|\int_{\mathbb{R}_{ \pm}}\left(\widehat{u}\left(t, \xi_{v}\right) e^{\frac{1}{4} i t \xi_{v}^{4}}-\widehat{u}(t, \xi) e^{\frac{1}{4} i t \xi^{4}}\right) \lambda^{-1} \overline{\chi_{1}\left(\lambda^{-1}\left(\xi-\xi_{v}\right), \lambda^{-1} \xi_{v}\right)} \mathrm{d} \xi\right| \\
& \quad \leq \int_{\mathbb{R}}\left|\xi-\xi_{v}\right| \int_{0}^{1}\left|\widehat{\mathcal{J} u}\left(t, \theta\left(\xi_{v}-\xi\right)+\xi\right)\right| \mathrm{d} \theta \cdot\left|\lambda^{-1} \chi_{1}\left(\lambda^{-1}\left(\xi-\xi_{v}\right), \lambda^{-1} \xi_{v}\right)\right| \mathrm{d} \xi \\
& \quad=|\lambda| \int_{\mathbb{R}} \int_{0}^{1}\left|\widehat{\mathcal{J} u}\left(t, \xi_{v}+\lambda \zeta(1-\theta)\right)\right| \mathrm{d} \theta\left|\zeta \chi_{1}\left(\zeta, \lambda^{-1} \xi_{v}\right)\right| \mathrm{d} \zeta .
\end{aligned}
$$

Since $\chi_{1}(\cdot, \alpha) \in \mathcal{S}(\mathbb{R})$ for $\alpha \geq 1$, it follows from (4.49), Hölder's inequality in $\zeta$, Minkowski's integral inequality, (4.1), and (2.9) that

$$
\begin{aligned}
& \left|\int_{\mathbb{R}_{ \pm}}\left(\widehat{u}\left(t, \xi_{v}\right) e^{\frac{1}{4} i t \xi_{v}^{4}}-\widehat{u}(t, \xi) e^{\frac{1}{4} i t \xi^{4}}\right) \lambda^{-1} \overline{\chi_{1}\left(\lambda^{-1}\left(\xi-\xi_{v}\right), \lambda^{-1} \xi_{v}\right)} \mathrm{d} \xi\right| \\
& \quad \lesssim|\lambda|^{\frac{1}{2}}\|\mathcal{J} u(t)\|_{L_{x}^{2}} \lesssim\left(t^{\frac{3}{4}}|v|\right)^{-\frac{1}{6}} \cdot t^{-\frac{1}{8}}\|u(t)\|_{\tilde{X}} .
\end{aligned}
$$

Hence, the $L^{\infty}$-estimate in (4.24) follows from (4.48) and (4.50).

For the $L^{2}$-estimate in the frequency space, we change variables using $\widetilde{\mathrm{v}}=\xi_{v}+$ $\lambda \zeta(1-\theta)$. Since

$$
\frac{\mathrm{d} \widetilde{\mathrm{v}}}{\mathrm{d} v}=\frac{1}{3} v^{-\frac{2}{3}}\left\{1-\zeta(1-\theta) t^{-\frac{1}{2}} v^{-\frac{2}{3}}\right\}
$$

(4.49), Minkowski’s integral inequality, (4.1), and (2.9) yield that

$$
\begin{aligned}
& \left\|\int_{\mathbb{R}_{ \pm}}\left(\widehat{u}\left(t, \xi_{v}\right) e^{\frac{1}{4} i t \xi_{v}^{4}}-\widehat{u}(t, \xi) e^{\frac{1}{4} i t \xi^{4}}\right) \lambda^{-1} \overline{\chi_{1}\left(\lambda^{-1}\left(\xi-\xi_{v}\right), \lambda^{-1} \xi_{v}\right)} \mathrm{d} \xi\right\|_{L_{v}^{2}(\Omega(t))} \\
& \lesssim \int_{0}^{1}\left\|\lambda \int_{\mathbb{R}}\left|\widehat{\mathcal{J} u}\left(t, \xi_{v}+\lambda \zeta(1-\theta)\right)\right|\left|\zeta \chi_{1}\left(\zeta, \lambda^{-1} \xi_{v}\right)\right| \mathrm{d} \zeta\right\|_{L_{v}^{2}(\Omega(t))} \mathrm{d} \theta \\
& \lesssim t^{-\frac{1}{2}}\|(\mathcal{J} u)(t, \widetilde{\mathrm{v}})\|_{L_{\widetilde{\mathrm{v}}}^{2}} \lesssim t^{-\frac{3}{8}} \cdot t^{-\frac{1}{8}}\|u(t)\|_{\tilde{X}} .
\end{aligned}
$$

Moreover, by (4.20), (4.4), and (2.9), we have

$$
\begin{aligned}
& \left\|\left(t^{\frac{3}{4}}|v|\right)^{-\frac{2}{3}} \widehat{u}\left(t, \xi_{v}\right)\right\|_{L_{v}^{2}(\Omega(t))}+\left\|\left(t^{\frac{3}{4}}|v|\right)^{-1}\right\|_{L_{v}^{2}(\Omega(t))} t^{-\frac{1}{8}}\|u(t)\|_{\tilde{X}} \\
& \lesssim t^{-\frac{1}{2}}\left\||\xi|^{-1} \widehat{u}(t, \xi)\right\|_{L_{\xi}^{2}\left(|\xi| \geq t^{-\frac{1}{4}}\right)}+t^{-\frac{3}{8}} \cdot t^{-\frac{1}{8}}\|u(t)\|_{\widetilde{X}} \\
& \lesssim t^{-\frac{3}{8}} \cdot t^{-\frac{1}{8}}\|u(t)\|_{\widetilde{X}} .
\end{aligned}
$$

Hence, the $L^{2}$-estimate in (4.24) follows from (4.45), (4.51), and (4.52). This concludes the proof. 


\section{Proof of the main theorem}

In this section, we prove Theorem 1. In Sect. 5.1, we derive an ordinary differential equation with respect to $\gamma$. In Sect. 5.2, we prove the global existence of the solution to (1.1). In Sect. 5.3, we show the asymptotic behavior of the global solution.

\subsection{ODE with respect to $\gamma$}

In this subsection, we prove the following proposition:

Proposition 4. Assume that $F$ satisfies (A-1) and (A-2). Let $u$ be a solution to (1.1) satisfying (1.26). Then, we have

$$
\left\|t\left(t^{\frac{3}{4}}|v|\right)^{\frac{1}{6}} \dot{\gamma}(t)\right\|_{L_{v}^{\infty}(\Omega(t))}+\left\|t^{\frac{11}{8}} \dot{\gamma}(t)\right\|_{L_{v}^{2}(\Omega(t))} \lesssim \varepsilon
$$

for $t \geq 1$, where the implicit constant is independent of $D$ and T. Here, $\gamma$ and $\Omega(t)$ are as in (1.28) and (4.20), respectively.

We use err to denote error terms that satisfy the estimates

$$
\left\|t\left(t^{\frac{3}{4}}|v|\right)^{\frac{1}{6}} \operatorname{err}\right\|_{L_{v}^{\infty}(\Omega(t))} \lesssim \varepsilon, \quad\left\|t^{\frac{11}{8}} \operatorname{err}\right\|_{L_{v}^{2}(\Omega(t))} \lesssim \varepsilon .
$$

Then, Proposition 4 says that

$$
\dot{\gamma}(t)=\text { err. }
$$

For the proof of Proposition 4, we use the following lemmas.

Lemma 9. For $t \geq 1, v \in \Omega(t)$, and $k=0,1,2$, we have

$$
t^{-1}|v|^{-\frac{k}{3}} \int_{\mathbb{R}}\left|\partial_{x}^{k} u^{\mathrm{ell}}(t, x) \chi(\lambda(x-v t))\right| \mathrm{d} x=\mathbf{e r r},
$$

where $\chi$ is a smooth function satisfying (4.3).

Proof. By (4.1), (3.25), and Lemma 2, we have

$$
\begin{aligned}
& t^{-1}|v|^{-\frac{k}{3}} \int_{\mathbb{R}}\left|\partial_{x}^{k} u^{\mathrm{ell}}(t, x) \chi(\lambda(x-v t))\right| \mathrm{d} x \\
& \quad \lesssim t^{-1} \cdot t^{-\frac{1}{4}}\left(t^{\frac{3}{4}}|v|\right)^{-\frac{5}{6}}|\lambda|^{-1} \sup _{x \in \mathbb{R}}\left|t^{\frac{k+1}{4}}\left\langle t^{-\frac{1}{4}} x\right\rangle^{-\frac{k}{3}+\frac{5}{6}} \partial_{x}^{k} u^{\mathrm{ell}}(t, x)\right| \\
& \quad \lesssim t^{-1}\left(t^{\frac{3}{4}}|v|\right)^{-\frac{1}{2}} \varepsilon .
\end{aligned}
$$

Moreover, it follows from Lemmas 7 and 2 with (3.23) that

$$
\begin{gathered}
\left\|t^{-1}|v|^{-\frac{k}{3}} \int_{\mathbb{R}}\left|\partial_{x}^{k} u^{\mathrm{ell}}(t, x) \chi(\lambda(x-v t))\right| \mathrm{d} x\right\|_{L_{v}^{2}(\Omega(t))} \\
\lesssim t^{-\frac{3}{2}}\left\|t^{\frac{k+1}{4}}\left\langle t^{-\frac{1}{4}} x\right\rangle^{-\frac{k}{3}+\frac{1}{3}} \partial_{x}^{k} u^{\mathrm{ell}}(t)\right\|_{L_{x}^{2}} \lesssim t^{-\frac{11}{8}} \varepsilon .
\end{gathered}
$$

We therefore obtain (5.2). 
Lemma 10. For $t \geq 1$ and $v \in \Omega(t)$, we have

$$
\left|\int_{\mathbb{R}}\left(u \overline{\mathcal{L} \Psi_{v}}\right)(t, x) \mathrm{d} x\right|=\mathbf{e r r},
$$

where $\mathcal{L}$ and $\Psi_{v}$ are as in (1.19) and (4.2), respectively.

Proof. Let $v \in \Omega(t)$ and let \pm be as in (4.8). From (4.16), (3.5), and (3.6), we have

$$
\begin{aligned}
\left(u \overline{\mathcal{L} \Psi_{v}}\right)(t, x)= & -i \frac{e^{-i \phi(t, x)}}{t \lambda} u^{\mathrm{hyp}, \pm}(t, x) \overline{\partial_{x} \tilde{\chi}(t, x)} \\
& -i \frac{e^{-i \phi(t, x)}}{t \lambda} u^{\mathrm{ell}}(t, x) \overline{\partial_{x} \widetilde{\chi}(t, x)} \\
& +O\left(|u(t, x)| t^{-1}\left(t^{\frac{3}{4}}|v|\right)^{-\frac{4}{3}}|\chi(\lambda(x-v t))|\right) \\
= & : E_{1}(t, x)+E_{2}(t, x)+E_{3}(t, x) .
\end{aligned}
$$

Note that $\tilde{\chi}_{0}$ defined in (4.18) has the same localization property as $\chi$. It follows from (5.4), (3.26), (4.1), (3.22), (4.17), and Lemma 2 that

$$
\begin{aligned}
\left|\int_{\mathbb{R}} E_{1}(t, x) \mathrm{d} x\right| & \lesssim t^{-\frac{13}{12}}\left(t^{\frac{3}{4}}|v|\right)^{\frac{1}{3}} \int_{\mathbb{R}}\left|\mathcal{J}_{ \pm} u^{\text {hyp }, \pm}(t, x) \tilde{\chi}(t, x)\right| \mathrm{d} x \\
& \lesssim t^{-\frac{5}{4}}\left(t^{\frac{3}{4}}|v|\right)^{-\frac{1}{3}}\left\||x|^{\frac{2}{3}} \mathcal{J}_{ \pm} u^{\mathrm{hyp}, \pm}(t)\right\|_{L_{x}^{2}}\|\tilde{\chi}(t)\|_{L_{x}^{2}} \\
& \lesssim t^{-1}\left(t^{\frac{3}{4}}|v|\right)^{-\frac{1}{6}} \varepsilon .
\end{aligned}
$$

In addition, we use Lemma 7, (4.17), (3.22), and Lemma 2 to obtain

$$
\begin{aligned}
\left\|\int_{\mathbb{R}} E_{1}(t, x) \mathrm{d} x\right\|_{L_{v}^{2}(\Omega(t))} & \lesssim\left\|t^{-\frac{13}{12}}\left(t^{\frac{3}{4}} v\right)^{\frac{1}{3}} \int_{\mathbb{R}}\left|\mathcal{J}_{ \pm} u^{\mathrm{hyp}, \pm}(t, x) \tilde{\chi}(t, x)\right| \mathrm{d} x\right\|_{L_{v}^{2}(\Omega(t))} \\
& \lesssim t^{-\frac{3}{2}}\left\||x|^{\frac{2}{3}} \mathcal{J}_{ \pm} u^{\mathrm{hyp}, \pm}\right\|_{L_{x}^{2}} \\
& \lesssim t^{-\frac{11}{8}} \varepsilon .
\end{aligned}
$$

From (5.4), (4.17), and Lemma 9, we have

$$
\left|\int_{\mathbb{R}} E_{2}(t, x) \mathrm{d} x\right| \lesssim t^{-1} \int_{\mathbb{R}}\left|u^{\mathrm{ell}}(t, x)\left(\partial_{x} \tilde{\chi}_{0}\right)\left(\lambda(x-v t), \lambda^{-1} \xi_{v}\right)\right| \mathrm{d} x=\mathbf{e r r} .
$$

Moreover, we use (5.4), (4.1), Proposition 2, and Lemma 2 to obtain

$$
\begin{aligned}
\left|\int_{\mathbb{R}} E_{3}(t, x) \mathrm{d} x\right| & \lesssim t^{-1}\left(t^{\frac{3}{4}}|v|\right)^{-\frac{4}{3}} \int_{\mathbb{R}}|u(t, x) \chi(\lambda(x-v t))| \mathrm{d} x \\
& \lesssim t^{-1}\left(t^{\frac{3}{4}}|v|\right)^{-\frac{3}{2}}|\lambda|^{-1}\left\|\left\langle t^{-\frac{1}{4}} x\right\rangle^{\frac{1}{6}} u(t)\right\|_{L_{x}^{\infty}} \\
& \lesssim t^{-1}\left(t^{\frac{3}{4}}|v|\right)^{-\frac{7}{6}} \varepsilon .
\end{aligned}
$$


In addition, (5.8) also yields that

$$
\left\|\int_{\mathbb{R}} E_{3}(t, x) \mathrm{d} x\right\|_{L_{v}^{2}(\Omega(t))} \lesssim t^{-1}\left\|\left(t^{\frac{3}{4}}|v|\right)^{-\frac{7}{6}}\right\|_{L_{v}^{2}(\Omega(t))} \varepsilon \lesssim t^{-\frac{11}{8}} \varepsilon .
$$

Hence, (5.3) follows from (5.4) and (5.9).

Finally, we prove Proposition 4.

Proof of Proposition 4. By (1.19), (1.1), and Lemma 10, we can write

$$
\begin{aligned}
\dot{\gamma}(t, v) & =-i \int_{\mathbb{R}}\left(\mathcal{L} u \cdot \overline{\Psi_{v}}\right)(t, x)+i \int_{\mathbb{R}}\left(u \overline{\mathcal{L} \Psi_{v}}\right)(t, x) \mathrm{d} x \\
& =\int_{\mathbb{R}} \partial_{x} F(u(t, x)) \overline{\Psi_{v}(t, x)} \mathrm{d} x+\text { err. }
\end{aligned}
$$

The bootstrap assumption (1.26), (A-1), (4.2), (4.1), and $\varepsilon \leq D^{-\frac{4}{3}}$ yield that

$$
\begin{aligned}
\left|\int_{\mathbb{R}} \partial_{x} F(u(t, x)) \overline{\Psi_{v}(t, x)} \mathrm{d} x\right| & \lesssim t^{-\frac{5}{4}}(D \varepsilon)^{4} \int_{\mathbb{R}}\left\langle t^{-\frac{1}{4}} x\right\rangle^{-1}\left|\Psi_{v}(t, x)\right| \mathrm{d} x \\
& \lesssim t^{-\frac{5}{4}}\left(t^{\frac{3}{4}}|v|\right)^{-1}(D \varepsilon)^{4}|\lambda|^{-1} \\
& \lesssim t^{-1}\left(t^{\frac{3}{4}}|v|\right)^{-\frac{2}{3}} \varepsilon .
\end{aligned}
$$

We therefore obtain (5.1). This concludes the proof of Proposition 4.

\subsection{Global existence}

In this subsection, by using Proposition 4, we prove the global existence of the solution to (1.1). From Proposition 1 and Lemma 1, this is reduced to showing (1.9), that is to say, to close the bootstrap estimate (1.26).

Let $C_{*}$ be as in (4.21). In the case $t^{-\frac{1}{4}}|x| \leq C_{*}$, Proposition 2 and Lemma 2 yield that

$$
\begin{aligned}
\left\|\left\langle t^{-\frac{1}{4}} x\right\rangle^{-\frac{k}{3}+\frac{1}{3}} \partial_{x}^{k} u(t)\right\|_{L_{x}^{\infty}\left(t^{-\frac{1}{4}}|x| \leq C_{*}\right)} & \lesssim\left\|\left\langle t^{-\frac{1}{4}} x\right\rangle^{-\frac{k}{3}+\frac{1}{6}} \partial_{x}^{k} u(t)\right\|_{L_{x}^{\infty}} \\
& \lesssim t^{-\frac{k+1}{4}-\frac{1}{8}}\|u(t)\|_{\tilde{X}} \lesssim \varepsilon t^{-\frac{k+1}{4}}
\end{aligned}
$$

for $k=0,1,2$. For the case $t^{-\frac{1}{4}}|x| \geq C_{*}$, owing to (4.22) and (4.1), it is reduced to showing that

$$
\|\gamma(t)\|_{L_{v}^{\infty}(\Omega(t))} \lesssim \varepsilon
$$

where $\Omega(t)$ is as in (4.20) and the implicit constant is independent of $D$ and $T$.

When $|v| \geq C_{*}, v \in \Omega(t)$ implies that $t \geq \max \left(1, C_{*}^{\frac{4}{3}}|v|^{-\frac{4}{3}}\right)$. Then, solving the ordinary differential equations in Proposition 4 with the initial time $t=1$, we have

$$
\gamma(t, v)=\gamma(1, v)+O\left(\varepsilon\left(t^{\frac{3}{4}}|v|\right)^{-\frac{1}{6}}\right) .
$$


It follows from (1.28), Lemma 5, the Gagliardo-Nirenberg inequality, (1.20), and Remark 4 that

$$
|\gamma(1, v)| \lesssim\|\widehat{u}(1)\|_{L_{\xi}^{\infty}}=\left\|e^{\frac{1}{4} i \xi^{4}} \widehat{u}(1)\right\|_{L_{\xi}^{\infty}} \lesssim\|u(1)\|_{L_{x}^{2}}^{\frac{1}{2}}\|\mathcal{J} u(1)\|_{L_{x}^{2}}^{\frac{1}{2}} \lesssim \varepsilon .
$$

By (5.11) and (5.12), we obtain (5.10) for $|v| \geq C_{*}$.

When $|v|<C_{*}$, let $t_{0}>1$ be $t_{0}:=C_{*}^{\frac{4}{3}}|v|^{-\frac{4}{3}}$. Then, solving the ordinary differential equations in Proposition 4 with the initial time $t=t_{0}$, we have

$$
\gamma(t, v)=\gamma\left(t_{0}, v\right)+O(\varepsilon) .
$$

Note that (4.7) and (4.4) yield that $N_{v} \sim|v|^{\frac{1}{3}} \sim t_{0}^{-\frac{1}{4}}$. Bernstein's inequality, Proposition 2, and Lemmas 6 and 2 with (2.9) yield that

$$
\begin{aligned}
\left|\gamma\left(t_{0}, v\right)\right| & \lesssim\left\|P_{N_{v}} u\left(t_{0}\right)\right\|_{L_{x}^{\infty}}\left\|\Psi_{v}\left(t_{0}\right)\right\|_{L_{x}^{1}}+\left\|u\left(t_{0}\right)\right\|_{L_{x}^{\infty}}\left\|\left(1-P_{N_{v}}\right) \Psi_{v}\left(t_{0}\right)\right\|_{L_{x}^{1}} \\
& \lesssim t_{0}^{\frac{1}{8}} \sum_{\substack{N \in 2^{\mathbb{Z}} \\
N \sim t_{0}^{-\frac{1}{4}}}}\left\|u_{N}\left(t_{0}\right)\right\|_{L_{x}^{2}}+t_{0}^{-\frac{1}{8}}\left\|u\left(t_{0}\right)\right\|_{\tilde{X}} \\
& \lesssim t_{0}^{-\frac{1}{8}}\left\|u\left(t_{0}\right)\right\|_{\tilde{X}} \lesssim \varepsilon .
\end{aligned}
$$

By (5.13) and (5.14), we obtain (5.10) for $|v|<C_{*}$. Accordingly, we conclude that (1.9) holds for any $t \in[1, T]$.

\subsection{Asymptotic behavior}

In this subsection, we present the proof of the asymptotic behavior of the global solution to (1.1).

Proposition 4 yields that there exists a unique function $W$ defined on $\mathbb{R} \backslash\{0\}$ such that for $t \geq 1$,

$$
\gamma(t, v)=\frac{1}{\sqrt{3}} W\left(\xi_{v}\right)+\widetilde{R}(t, v)
$$

where

$$
\left\|\left(t^{\frac{3}{4}}|v|\right)^{\frac{1}{6}} \widetilde{R}(t, v)\right\|_{L_{v}^{\infty}(\Omega(t))}+\left\|t^{\frac{3}{8}} \widetilde{R}(t, v)\right\|_{L_{v}^{2}(\Omega(t))} \lesssim \varepsilon
$$

We extend $W$ to $\mathbb{R}$ by defining

$$
W(0)=\int_{\mathbb{R}} u_{0}(x) \mathrm{d} x .
$$

Then, by (5.10), we have

$$
\|W\|_{L_{\xi_{v}}^{\infty}} \leq \varepsilon
$$

Moreover, changing variable $v=\xi_{v}$ defined in (4.4) and Lemma 7 with (4.20) yield that

$$
\|\gamma(t, v)\|_{L_{\xi_{v}}^{2}\left(\left|\xi_{v}\right| \geq C_{*}^{\frac{1}{3}} t^{-\frac{1}{4}}\right)}=\left\|v^{-\frac{1}{3}} \gamma(t, v)\right\|_{L_{v}^{2}(\Omega(t))} \lesssim\|u(t)\|_{L_{x}^{2}}
$$


In particular, by (1.25), we have

$$
\|\gamma(1, v)\|_{L_{\xi_{v}}^{2}\left(\left|\xi_{v}\right| \geq C_{*}^{\frac{1}{3}}\right)} \lesssim \varepsilon .
$$

Then, it follows from (5.16), (5.15), and (5.17) that

$$
\|W\|_{L_{\xi_{v}}^{2}} \leq\|W\|_{L_{\xi_{v}}^{2}\left(\left|\xi_{v}\right| \leq C_{*}^{\frac{1}{3}}\right)}+\|W\|_{L_{\xi_{v}}^{2}\left(\left|\xi_{v}\right| \geq C_{*}^{\frac{1}{3}}\right)} \lesssim \varepsilon .
$$

By (5.15) and Proposition 3, we obtain the asymptotic behavior (1.13) and (1.14).

Finally, we show the existence of the self-similar solution and the asymptotic behavior in the self-similar region $\mathfrak{X}^{\text {self }}(t)$. We use the self-similar change of variables (2.13). Let $\rho>0$ be a constant specified later and let $C \gg 1$. By choosing $C$ sufficiently large and (3.3), we have

$$
P_{\geq C t^{\rho-\frac{1}{4}}} u_{N}(t, x)=P_{\geq C t^{\rho-\frac{1}{4}}} u_{N}^{\mathrm{ell}}(t, x)
$$

for $|x| \lesssim t^{3 \rho}$. We set $\mathfrak{Y}^{0}(t):=\left\{y \in \mathbb{R}:|y| \lesssim t^{3 \rho}\right\}$.

From Bernstein's inequality, (2.14), (5.18), (3.13), and Lemmas 1 and 2, we have

$$
\begin{aligned}
\left\|\partial_{t} P_{\leq C t^{\rho}} U(t)\right\|_{L_{y}^{\infty}\left(\mathfrak{Y}^{0}(t)\right)} & \lesssim t^{\frac{\rho}{2}}\left\|\partial_{t} P_{\leq C t^{\rho}} U(t)\right\|_{L_{y}^{2}\left(\mathfrak{Y}^{0}(t)\right)} \\
& \lesssim t^{\frac{3}{2} \rho-\frac{9}{8}}\|\Lambda u(t)\|_{L_{x}^{2}}+t^{\frac{\rho}{2}-\frac{7}{8}} \sum_{\substack{N \in \mathbb{Z}^{\mathbb{Z}} \\
N \sim t^{\rho-\frac{1}{4}}}}\left\|u_{N}^{\mathrm{ell}}(t)\right\|_{L_{x}^{2}} \\
& \lesssim \varepsilon t^{-1-\min \left(-\frac{3}{2} \rho+\frac{1}{8}-\varepsilon, \frac{5}{2} \rho\right)} .
\end{aligned}
$$

Furthermore, (5.18), (3.9), (3.13), and Lemma 2 yield

$$
\begin{aligned}
& \left\|P_{>C t^{\rho}} U(t)\right\|_{L_{y}^{\infty}\left(\mathfrak{Y}^{0}(t)\right)} \lesssim t^{\frac{1}{4}}\left(\sum_{N \in 2^{\mathbb{Z}}} N\left\|u_{N}^{\mathrm{ell}}(t)\right\|_{L_{x}^{2}}^{2}\right)^{\frac{1}{2}} \\
& N>C t^{\rho-\frac{1}{4}} \\
& +t^{\frac{1}{4}} \sum_{N \in 2^{\mathbb{Z}}}\left\|\left(1-P_{\frac{N}{2} \leq-\leq 2 N}\right)\left|\partial_{x}\right|^{\frac{1}{2}} u_{N}^{\mathrm{ell}}(t)\right\|_{L_{x}^{2}} \\
& N>C t^{\rho-\frac{1}{4}} \\
& \lesssim t^{-\frac{5}{2} \rho} \varepsilon, \\
& \left\|P_{>C t^{\rho}} U(t)\right\|_{L_{y}^{2}\left(\mathfrak{Y}^{0}(t)\right)} \lesssim t^{\frac{1}{8}}\left(\sum_{N \in 2^{\mathbb{Z}}}\left\|u_{N}^{\mathrm{ell}}(t)\right\|_{L_{x}^{2}}^{2}\right)^{\frac{1}{2}} \\
& N>C t^{\rho-\frac{1}{4}} \\
& +t^{\frac{1}{8}} \sum_{\substack{N \in 2^{\mathbb{Z}} \\
N>C t^{\rho-\frac{1}{4}}}}\left\|\left(1-P_{\frac{N}{2} \leq \cdot \leq 2 N}\right) u_{N}^{\mathrm{ell}}(t)\right\|_{L_{x}^{2}} \\
& \lesssim t^{-3 \rho} \varepsilon .
\end{aligned}
$$


By setting $\rho:=\frac{1}{4}\left(\frac{1}{8}-\varepsilon\right)$ with (5.19)-(5.21), there exists $Q \in L_{y}^{\infty}(\mathbb{R})$ such that

$$
\|U(t)-Q\|_{L_{y}^{\infty}\left(\mathfrak{Y}^{0}(t)\right)} \lesssim \varepsilon t^{-\frac{5}{2} \rho}, \quad\|U(t)-Q\|_{L_{y}^{2}\left(\mathfrak{Y}^{0}(t)\right)} \lesssim \varepsilon t^{-3 \rho} .
$$

Moreover, it follows from (5.22), (2.13), and (1.9) that

$$
\left\|\langle\cdot\rangle^{\frac{1}{3}} Q\right\|_{L_{y}^{\infty}} \leq \lim _{t \rightarrow \infty}\left(t^{\rho}\|Q-U(t)\|_{L_{y}^{\infty}\left(\mathfrak{Y}^{0}(t)\right)}+\left\|\langle\cdot\rangle^{\frac{1}{3}} U(t)\right\|_{L_{y}^{\infty}}\right) \lesssim \varepsilon .
$$

By (1.22) and (1.1), we have

$$
\begin{aligned}
\Lambda u(t, x) & =4 t \partial_{x}^{-1} \partial_{t} u(t, x)+x u(t, x) \\
& =-i t \partial_{x}^{3} u(t, x)+4 t F(u(t, x))+x u(t, x) .
\end{aligned}
$$

It follows from (5.23), (2.13), and Lemma 1 that

$$
\left\|\partial_{y}^{3} U(t)+i y U(t)+4 i F(U(t))\right\|_{L_{y}^{2}}=\left\|(\Lambda u)\left(t, t^{\frac{1}{4}} y\right)\right\|_{L_{y}^{2}} \lesssim t^{\varepsilon-\frac{1}{8}} .
$$

By taking the limit as $t \rightarrow \infty, Q$ solves (1.10). In addition, (5.22), and (1.4) yield that

$$
\begin{aligned}
\int_{\mathbb{R}} Q(y) \mathrm{d} y & =\lim _{t \rightarrow \infty} \int_{-t^{\rho}}^{t^{\rho}} Q(y) \mathrm{d} y=\lim _{t \rightarrow \infty} \int_{-t^{\rho}}^{t^{\rho}} U(t, y) \mathrm{d} y \\
& =\lim _{t \rightarrow \infty} \int_{\mathbb{R}} U(t, y) \mathrm{d} y=\int_{\mathbb{R}} u_{0}(x) \mathrm{d} x .
\end{aligned}
$$

By (1.10) and (5.24), $\mathfrak{u}(t, x):=t^{-\frac{1}{4}} Q\left(t^{-\frac{1}{4}} x\right)$ solves (1.1) with $\mathfrak{u}(0)=\int_{\mathbb{R}} u_{0}(x) \mathrm{d} x \delta_{0}$, where $\delta_{0}$ is the Dirac delta measure concentrated at the origin. Moreover, (1.11) and (1.12) follow from (5.22) and (2.13), which concludes the proof of Theorem 1.

\section{Acknowledgements}

This work was supported by JSPS KAKENHI Grant Numbers JP19K14578 and JP20K14342. The authors would like to thank the anonymous referees for helpful comments.

Open Access. This article is licensed under a Creative Commons Attribution 4.0 International License, which permits use, sharing, adaptation, distribution and reproduction in any medium or format, as long as you give appropriate credit to the original author(s) and the source, provide a link to the Creative Commons licence, and indicate if changes were made. The images or other third party material in this article are included in the article's Creative Commons licence, unless indicated otherwise in a credit line to the material. If material is not included in the article's Creative Commons licence and your intended use is not permitted by statutory regulation or exceeds the permitted use, you will need to obtain permission directly from the copyright holder. To view a copy of this licence, visit http://creativecommons.org/licenses/ by/4.0/.

Publisher's Note Springer Nature remains neutral with regard to jurisdictional claims in published maps and institutional affiliations. 


\section{REFERENCES}

[1] K. Aoki, N. Hayashi, and P. I. Naumkin, Global existence of small solutions for the fourth-order nonlinear Schrödinger equation, NoDEA Nonlinear Differential Equations Appl. 23 (2016), no. 6, Art. 65, 18 pp.

[2] M. Ben-Artzi, H. Koch, and J.-C. Saut, Dispersion estimates for fourth order Schrödinger equations, C. R. Acad. Sci. Paris Sér. I Math. 330 (2000), no. 2, 87-92.

[3] B. Harrop-Griffiths, Long time behavior of solutions to the $m K d V$, Comm. Partial Differential Equations 41 (2016), no. 2, 282-317.

[4] B. Harrop-Griffiths, M. Ifrim, and D. Tataru, The lifespan of small data solutions to the KP-I, Int. Math. Res. Not. IMRN 2017, no. 1, 1-28.

[5] N. Hayashi and P. I. Naumkin, Factorization technique for the fourth-order nonlinear Schrödinger equation, Z. Angew. Math. Phys. 66 (2015), no. 5, 2343-2377.

[6] N. Hayashi and P. I. Naumkin, Global existence and asymptotic behavior of solutions to the fourthorder nonlinear Schrödinger equation in the critical case, Nonlinear Anal. 116 (2015), 112-131.

[7] N. Hayashi and P. I. Naumkin, Large time asymptotics for the fourth-order nonlinear Schrödinger equation, J. Differential Equations 258 (2015), no. 3, 880-905.

[8] N. Hayashi and P. I. Naumkin, On the inhomogeneous fourth-order nonlinear Schrödinger equation J. Math. Phys. 56 (2015), no. 9, 093502, 25 pp.

[9] N. Hayashi and P. I. Naumkin, Higher-order nonlinear Schrödinger equation in $2 D$ case, Tohoku Math. J. (2) 72 (2020), no. 1, 15-37.

[10] N. Hayashi, J. A. Mendez-Navarro, and P. I. Naumkin, Scattering of solutions to the fourth-order nonlinear Schrödinger equation, Commun. Contemp. Math. 18 (2016), no. 3, 1550035, 24 pp.

[11] N. Hayashi, J. A. Mendez-Navarro, and P. I. Naumkin, Asymptotics for the fourth-order nonlinear Schrödinger equation in the critical case, J. Differential Equations 261 (2016), no. 9, 5144-5179.

[12] H. Hirayama and M. Okamoto, Well-posedness and scattering for fourth order nonlinear Schrödinger type equation at the scaling critical regularity, Comm. Pure Appl. Anal. 15 (2016), no. 3, 831-851.

[13] M. Ifrim and D. Tataru, Global bounds for the cubic nonlinear Schrödinger equation (NLS) in one space dimension, Nonlinearity 28 (2015), no. 8, 2661-2675.

[14] B. Juarez-Campos and P. I. Naumkin, Large time asymptotics for the higher-order nonlinear nonlocal Schrödinger equation, Nonlinear Anal. 205 (2021), 112238, 26 pp.

[15] P. I. Naumkin and I. Sánchez-Suárez, KdV type asymptotics for solutions to higher-order nonlinear Schrödinger equations, Electron. J. Differential Equations 2020, Paper No. 77, 34 pp.

[16] M. Okamoto, Large time asymptotics of solutions to the short-pulse equation, NoDEA Nonlinear Differential Equations Appl. 24 (2017), no. 4, 24:42.

[17] M. Okamoto, Long-time behavior of solutions to the fifth-order modified KdV-type equation, Adv. Differential Equations 23 (2018), no. 9-10, 751-792.

[18] M. Okamoto, Asymptotic behavior of solutions to a higher-order KDV-type equation with critical nonlinearity, Evol. Equ. Control Theory 8 (2019), no. 3, 567-601.

[19] J.-C. Saut and J. Segata, Asymptotic behavior in time of solution to the nonlinear Schrödinger equation with higher order anisotropic dispersion, Discrete Contin. Dyn. Syst. 39 (2019), no. 1, 219-239. 
Mamoru Okamoto

Department of Mathematics, Graduate School of Science Osaka University

Toyonaka

Osaka 560-0043

Japan

E-mail: okamoto@math.sci.osaka-u.ac.jp

Kota Uriya

Department of Applied Mathematics, Faculty of Science Okayama University of Science

Okayama 700-0005

Japan

Accepted: 14 July 2021 